\title{
Counting walks in a quadrant: a unified approach via boundary value problems
}

Received March 10, 2010 and in revised form January 3, 2011 and February 13, 2011

\begin{abstract}
The aim of this article is to introduce a unified method to obtain explicit integral representations of the trivariate generating function counting the walks with small steps which are confined to a quarter plane. For many models, this yields for the first time an explicit expression of the counting generating function. Moreover, the nature of the integrand of the integral formulas is shown to be directly dependent on the finiteness of a naturally attached group of birational transformations as well as on the sign of the covariance of the walk.
\end{abstract}

Keywords. Lattice walk, counting generating function, boundary value problem, conformal mapping, Weierstrass elliptic function, Riemann surface, uniformization

\section{Introduction}

The enumeration of planar lattice walks is a classical topic in combinatorics. For a given set $\mathcal{S}$ of steps, it is a matter of counting the numbers of paths of a certain length, having jumps in $\mathcal{S}$, starting and ending at some arbitrary points, and possibly restricted to a region of the plane. There are two main questions:

- How many such paths exist?

- Is the underlying generating function rational, algebraic, holonomic (i.e., a solution of a linear differential equation with polynomial coefficients) or non-holonomic?

For instance, if the paths are not restricted to a region, or if they are constrained to a half-plane, the counting generating function can be made explicit and is rational or algebraic [7], respectively. It is then natural to consider the walks confined to the intersection of two half-planes, as the quadrant $\mathbb{Z}_{+}^{2}$. The situation seems richer: some walks have an algebraic counting function (see $[13,14]$ for the walk with unit step set $\mathcal{S}=\{\mathrm{W}, \mathrm{NE}, \mathrm{S}\}$ ), while others have a counting function that is not even holonomic (see [7] for the knight walk). Henceforth, we focus on these walks staying in $\mathbb{Z}_{+}^{2}$.

Bousquet-Mélou and Mishna have recently [6] initiated a systematic study of the walks confined to $\mathbb{Z}_{+}^{2}$, starting at the origin and having small steps, which means that

K. Raschel: CNRS and Université de Tours, Laboratoire de Mathématiques et Physique Théorique, Parc de Grandmont, 37200 Tours, France; e-mail: Kilian.Raschel@1mpt.univ-tours.fr

Mathematics Subject Classification (2010): Primary 05A15; Secondary 30F10, 30D05 
the set of admissible steps $\mathcal{S}$ is included in the set of the eight nearest neighbors, i.e.,

$$
\mathcal{S} \subset\{\mathrm{W}, \mathrm{NW}, \mathrm{N}, \mathrm{NE}, \mathrm{E}, \mathrm{SE}, \mathrm{S}, \mathrm{SW}\}{ }^{1}
$$

On the boundary, the jumps are the natural ones: the steps that would take the walk out $\mathbb{Z}_{+}^{2}$ are discarded. There are $2^{8}$ such models. Among these, there are obvious symmetries and Bousquet-Mélou and Mishna [6] show that there are in fact 79 types of essentially distinct walks-we will often refer to these 79 walks tabulated in [6].

The central object for the study of these 79 walks is the following: denoting by $q(i, j ; n)$ the number of paths confined to $\mathbb{Z}_{+}^{2}$, having length $n$, starting at $(0,0)$ and ending at $(i, j)$, their generating function is defined as

$$
Q(x, y ; z)=\sum_{i, j, n \geq 0} q(i, j ; n) x^{i} y^{j} z^{n} .
$$

Proposition 1. We have

$$
\begin{aligned}
x y z\left[\sum_{(i, j) \in \mathcal{S}} x^{i} y^{j}\right. & -1 / z] Q(x, y ; z) \\
& =c(x ; z) Q(x, 0 ; z)+\widetilde{c}(y ; z) Q(0, y ; z)-z \delta Q(0,0 ; z)-x y,
\end{aligned}
$$

where we have denoted

$$
c(x ; z)=z x \sum_{(i,-1) \in \mathcal{S}} x^{i}, \quad \widetilde{c}(y ; z)=z y \sum_{(-1, j) \in \mathcal{S}} y^{j}, \quad \delta= \begin{cases}1 & \text { if } \mathrm{SW} \in \mathcal{S}, \\ 0 & \text { if } \mathrm{SW} \notin \mathcal{S} .\end{cases}
$$

This functional equation is the fundamental starting point of our study —and is also so for almost all other works on the topic. It relates the trivariate generating function $Q(x, y ; z)$ to the bi- and univariate generating functions $Q(x, 0 ; z), Q(0, y ; z)$ and $Q(0,0 ; z)$ counting the walks which end on the borders. Notice that (2) simply follows from the step by step construction of the walks; its proof may be found in [6, Section 4]. The polynomial

$$
x y z\left[\sum_{(i, j) \in \mathcal{S}} x^{i} y^{j}-1 / z\right]
$$

appearing in (2) is usually called the kernel of the walk. If $k$ is the cardinality of $\mathcal{S}$, then (2) is valid at least on $\{|x| \leq 1,|y| \leq 1,|z|<1 / k\}$, since clearly $q(i, j ; n) \leq k^{n}$.

In this way, to answer both questions stated at the beginning of this paper, it suffices to solve (2). A key idea then is to consider a certain group, introduced in [20] in a probabilistic context, and called the group of the walk. This group of birational transformations of $\mathbb{C}^{2}$, which leaves invariant the step generating function $\sum_{(i, j) \in \mathcal{S}} x^{i} y^{j}$, is the group $W=\langle\Psi, \Phi\rangle$ generated by

$$
\Psi(x, y)=\left(x, \frac{\sum_{(i,-1) \in \mathcal{S}} x^{i}}{\sum_{(i,+1) \in \mathcal{S}} x^{i}} \frac{1}{y}\right), \quad \Phi(x, y)=\left(\frac{\sum_{(-1, j) \in \mathcal{S}} y^{j}}{\sum_{(+1, j) \in \mathcal{S}} y^{j}} \frac{1}{x}, y\right) .
$$

\footnotetext{
1 For the step set $\mathcal{S}$, we shall equally denote it by using the cardinal points $\{\mathrm{W}, \mathrm{NW}, \mathrm{N}, \mathrm{NE}, \mathrm{E}$, SE, S, SW $\}$ or the cartesian points $\{-1,0,1\}^{2} \backslash\{(0,0)\}$.
} 

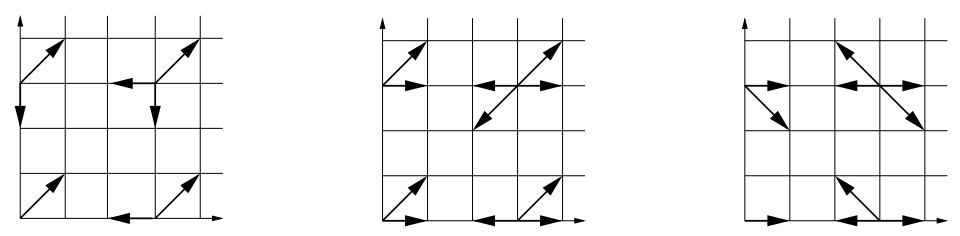

Fig. 1. Three famous examples, known as Kreweras', Gessel's and Gouyou-Beauchamps' walks. They have been, and are still, the object of many studies (see, e.g., $[5,12,13],[4,16,17]$ and $[8$, 23], respectively).

Obviously $\Psi \circ \Psi=\Phi \circ \Phi=\mathrm{id}$, and $W$ is a dihedral group—of order even and at least four. This order is calculated in [6] for each of the 79 cases: 23 walks admit a finite group (of order four, six or eight), and the 56 others have an infinite group.

For the 23 walks with a finite group, the answers to both questions (regarding explicit expression and nature of the function (1)) have been given recently. Indeed, the article [6] successfully treats 22 of the 23 models associated with a finite group: the series (1) is made explicit and is shown to be either algebraic or transcendental but holonomic. As for the 23rd walk (namely, Gessel's walk represented in Figure 1), Bostan and Kauers [4] have given a computer-aided proof of the algebraicity of the function (1). Furthermore, using a powerful computer algebra system, they have specified the minimal polynomials. Thanks to these polynomials, van Hoeij [4, Appendix] has managed to express the function (1) by radicals. At the same time, we gave in [17] an explicit integral representation of (1) for Gessel's walk, this time without computer help. Based on ideas of [9, Chapter 4], alternative proofs for the nature of (1) for these 23 cases are given in [10]. Moreover, in the work in preparation [2], Bostan et al. obtain integral representations of the function (1) for the 23 walks having a finite group, by using a mathematical and algorithmic method, based on creative telescoping and on the resolution of differential equations of order two in terms of hypergeometric functions.
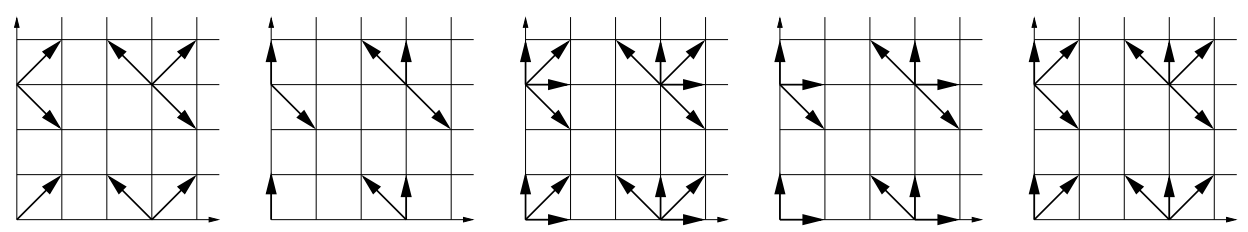

Fig. 2. The five singular walks in the classification of [6].

Concerning the 56 walks with an infinite group, the five represented in Figure 2 are special; they are called singular. Of these, two cases are solved: in [21], Mishna and Rechnitzer have considered the walks with step sets $\mathcal{S}=\{\mathrm{NW}, \mathrm{NE}, \mathrm{SE}\}$ and $\{\mathrm{NW}, \mathrm{N}, \mathrm{SE}\}$, have given the series (1) explicitly, and have shown that it is non-holonomic. What is left is 54 walks whose status is currently unsettled, as regards an explicit expression as well as holonomicity. However, certain asymptotic conjectures are proposed in [3]. 


\section{Main results}

The aim of this article is to introduce a unified approach giving an explicit expression of the generating function (1) for any of the 79 walks. We start with the non-singular walks. Note that important notations appear below the statement.

Theorem 1. Assume that the walk is one of the 74 non-singular walks.

(i) The following integral representations involving $Q(x, 0 ; z)$ and $Q(0, y ; z)$ hold:

$$
\begin{aligned}
& c(x ; z) Q(x, 0 ; z)-c(0 ; z) Q(0,0 ; z)=x Y_{0}(x ; z) \\
& \quad+\frac{1}{2 \imath \pi} \int_{x_{1}(z)}^{x_{2}(z)}\left[Y_{0}(t ; z)-Y_{1}(t ; z)\right]\left[\frac{\partial_{t} w(t ; z)}{w(t ; z)-w(x ; z)}-\frac{\partial_{t} w(t ; z)}{w(t ; z)-w(0 ; z)}\right] d t, \\
& \widetilde{c}(y ; z) Q(0, y ; z)-\widetilde{c}(0 ; z) Q(0,0 ; z)=X_{0}(y ; z) y \\
& \quad+\frac{1}{2 \imath \pi} \int_{y_{1}(z)}^{y_{2}(z)}\left[X_{0}(t ; z)-X_{1}(t ; z)\right]\left[\frac{\partial_{t} \widetilde{w}(t ; z)}{\widetilde{w}(t ; z)-\widetilde{w}(y ; z)}-\frac{\partial_{t} \widetilde{w}(t ; z)}{\widetilde{w}(t ; z)-\widetilde{w}(0 ; z)}\right] d t .
\end{aligned}
$$

(ii) The value of $Q(0,0 ; z)$ is determined as follows.

- If SW $\in \mathcal{S},{ }^{2}$ then for any $\left(x_{0}, y_{0}, z_{0}\right) \in\{|x| \leq 1,|y| \leq 1,|z|<1 / k\}$ at which the kernel (4) vanishes, we have

$$
\begin{aligned}
Q(0,0 ; z)=x_{0} y_{0} / z & -\left[c\left(x_{0} ; z\right) Q\left(x_{0}, 0 ; z\right)-c(0 ; z) Q(0,0 ; z)\right] / z \\
- & {\left[\widetilde{c}\left(y_{0} ; z\right) Q\left(0, y_{0} ; z\right)-\widetilde{c}(0 ; z) Q(0,0 ; z)\right] / z . }
\end{aligned}
$$

- If SW $\notin \mathcal{S}$, then

$$
Q(0,0 ; z)=\lim _{x \rightarrow 0} \frac{c(x ; z) Q(x, 0 ; z)-c(0 ; z) Q(x, 0 ; z)}{c(x ; z)} .
$$

(iii) The function $Q(x, y ; z)$ has the explicit expression

$$
Q(x, y ; z)=\frac{c(x ; z) Q(x, 0 ; z)+\widetilde{c}(y ; z) Q(0, y ; z)-z \delta Q(0,0 ; z)-x y}{x y z\left[\sum_{(i, j) \in \mathcal{S}} x^{i} y^{j}-1 / z\right]} .
$$

In the statement of Theorem 1:

- $c$ and $\tilde{c}$ are defined in (3).

- $Y_{0}$ and $Y_{1}$ (resp. $X_{0}$ and $X_{1}$ ) are the $y$ - (resp. $x$-) roots of the kernel (4), which is a second-degree polynomial. They are chosen such that $\left|Y_{0}\right| \leq\left|Y_{1}\right|\left(\right.$ resp. $\left.\left|X_{0}\right| \leq\left|X_{1}\right|\right)$ (see Lemma 1). Their expression is given in (9) (resp. (10)).

- $x_{1}(z)$ and $x_{2}(z)$ (resp. $y_{1}(z)$ and $y_{2}(z)$ ) are two of the four branch points of $Y_{0}$ and $Y_{1}$ (resp. $X_{0}$ and $X_{1}$ ) (see Subsection 3.2, below (8), for their proper definition). These four branch points can be characterized as the only points satisfying $Y_{0}=Y_{1}$ (resp. $\left.X_{0}=X_{1}\right)$. An equivalent definition is that they are the roots of the discriminant of the kernel (8) viewed as a second-degree polynomial in the variable $y$ (resp. $x$ ) (see (7)).

\footnotetext{
2 This condition is equivalent to $\delta=1$ in (2), and to $c(0 ; z)=\widetilde{c}(0 ; z)=z$ (see (3)).
} 
$-w$ and $\widetilde{w}$ are conformal mappings with additional gluing properties. While it is easy to show their existence (see Subsection 3.3), finding their expression is, generally speaking, quite a difficult task. Theorem 6 of this paper, which gives explicit formulas for $w$ and $\widetilde{w}$, is one of our main contributions. Because of notation, we prefer stating it in Section 6 rather than here: the expressions of $w$ and $\widetilde{w}$ we obtain indeed strongly involve $\wp$-Weierstrass elliptic functions, and the latter naturally appear due to the uniformization (in Section 5) of the set given by the zeros of the kernel (4), generically isomorphic to a Riemann surface of genus 1 . In Theorems 2 and 3 below, we give important and complementary specifications to Theorem 6.

For the 51 non-singular walks with an infinite group, the function $Q(x, y ; z)$ is made explicit for the first time- to the best of our knowledge. After the work [17] on Gessel's walk, this paper also provides integral representations of $Q(x, y ; z)$ for the 22 other walks admitting a finite group. As we are going to see now, it turns out that the finiteness of the group actually directly influences the nature (rational, algebraic, holonomic, nonholonomic) of the functions $w$ and $\widetilde{w}$ present in these integral representations. Another important quantity happens to be (the sign of) the covariance of $\mathcal{S}$, namely,

$$
\sum_{(i, j) \in \mathcal{S}} i j .
$$

Theorem 2. If the group of the walk is finite (resp. infinite), then $w$ and $\widetilde{w}$ are algebraic (resp. non-holonomic, and then, of course, non-algebraic). Furthermore, in the case of a finite group, if in addition the covariance (6) of the walk is negative or zero (resp. positive), then $w$ and $\widetilde{w}$ are rational (resp. algebraic non-rational).

Theorem 2 is summarized in Figure 6: in particular, in the finite group case, we compare a posteriori the nature of $w$ and $\widetilde{w}$ with that of $Q(x, y ; z)$, known from $[4,6,10]$. If the group is finite, Theorem 3 below goes much further than Theorem 2: in the rational (resp. algebraic) case, it provides the rational expressions (resp. the minimal polynomials) of $w$ and $\widetilde{w}$. For its statement we need the notations:

$-x_{3}(z)$ and $x_{4}(z)$ (resp. $y_{3}(z)$ and $\left.y_{4}(z)\right)$ are the remaining branch points of $Y_{0}$ and $Y_{1}$ (resp. $X_{0}$ and $X_{1}$ ) (see again Subsection 3.2, below (8), for their exact definition).

Theorem 3. In the finite group case, the explicit expressions of $w$ and $\widetilde{w}$ are as follows.

(i) If the walk is associated with a group of order four, then $w$ and $\widetilde{w}$ are affine combinations of, respectively,

$$
\frac{\left[t-x_{1}(z)\right]\left[t-x_{4}(z)\right]}{\left[t-x_{2}(z)\right]\left[t-x_{3}(z)\right]}, \quad \frac{\left[t-y_{1}(z)\right]\left[t-y_{4}(z)\right]}{\left[t-y_{2}(z)\right]\left[t-y_{3}(z)\right]} .
$$

(ii) For both walks $\{\mathrm{N}, \mathrm{SE}, \mathrm{W}\}$ and $\{\mathrm{N}, \mathrm{E}, \mathrm{SE}, \mathrm{S}, \mathrm{W}, \mathrm{NW}\}$, $w$ and $\widetilde{w}$ are affine combinations of, respectively,

$$
\begin{aligned}
& \frac{u(t)}{\left[t-x_{2}(z)\right]\left[t-1 / x_{2}(z)^{1 / 2}\right]^{2}}, \quad \frac{\widetilde{u}(t)}{\left[t-y_{2}(z)\right]\left[t-1 / y_{2}(z)^{1 / 2}\right]^{2}}, \\
& \text { with } u(t)=t^{2} \text { and } \widetilde{u}(t)=t(\operatorname{resp} . u(t)=\widetilde{u}(t)=t(t+1)) \text { for }\{\mathrm{N}, \mathrm{SW}, \mathrm{E}\}(\text { resp. } \\
& \{\mathrm{N}, \mathrm{W}, \mathrm{SW}, \mathrm{S}, \mathrm{E}, \mathrm{NE}\} \text { ). }
\end{aligned}
$$


(iii) For each of the three walks $\{\mathrm{NE}, \mathrm{S}, \mathrm{W}\},\{\mathrm{N}, \mathrm{E}, \mathrm{SW}\}$ and $\{\mathrm{N}, \mathrm{NE}, \mathrm{E}, \mathrm{S}, \mathrm{SW}, \mathrm{W}\}$, there exist $\alpha(z), \beta(z), \delta(z), \gamma(z)$ which are algebraic with respect to $z$-and made explicit in the proof-such that $w=\widetilde{w}$ is the only root with a pole at $x_{2}(z)$ of

$$
w^{2}+\left[\alpha(z)+\frac{\beta(z) u(t)}{\left[t-x_{2}(z)\right]\left[t-1 / x_{2}(z)^{1 / 2}\right]^{2}}\right] w+\left[\delta(z)+\frac{\gamma(z) u(t)}{\left[t-x_{2}(z)\right]\left[t-1 / x_{2}(z)^{1 / 2}\right]^{2}}\right],
$$

with $u(t)=t^{2}($ resp. $u(t)=t, u(t)=t(t+1))$ for $\{\mathrm{NE}, \mathrm{S}, \mathrm{W}\}$ (resp. \{N, E, SW\}, $\{N, N E, E, S, S W, W\})$.

(iv) For the walk $\{\mathrm{E}, \mathrm{SE}, \mathrm{W}, \mathrm{NW}\}, w$ and $\widetilde{w}$ are affine combinations of, respectively,

$$
\frac{t^{2}}{\left[t-x_{2}(z)\right][t-1]^{2}\left[t-x_{3}(z)\right]}, \quad \frac{t(t+1)^{2}}{\left[t-x_{2}(z)\right]^{2}\left[t-x_{3}(z)\right]^{2}} .
$$

We emphasize that it is not necessary to know the affine combinations appearing in (i), (ii) and (iv) above, since in Theorem $1, w$ and $\widetilde{w}$ only appear through $\partial_{t} w(t ; z) /[w(t ; z)-$ $w(x ; z)]$ and $\partial_{t} \widetilde{w}(t ; z) /[\widetilde{w}(t ; z)-\widetilde{w}(y ; z)]$.

Together with Gessel's walk $\{\mathrm{E}, \mathrm{SW}, \mathrm{W}, \mathrm{NE}\}$, for which the functions $w$ and $\widetilde{w}$ have been found in [17], Theorem 3 gives the simplified expression of these functions in all finite group cases (see the tables in [6] or Figure 6 in this paper).

Thanks to Theorems 3 and 6 , which give formulas for $w$ and $\widetilde{w}$, Theorem 1 settles the problem of making explicit the numbers of walks $q(i, j ; n)$. The function $Q(x, y ; z)$ being indeed found (at least) within the domain $\{|x| \leq 1,|y| \leq 1, z \in] 0,1 / k[\}$, its coefficients $\sum_{n \geq 0} q(i, j ; n) z^{n}$ can very easily be obtained from the Cauchy formulas, for any $z \in] 0,1 / k\left[\right.$. Since the radius of convergence of the series $\sum_{n \geq 0} q(i, j ; n) z^{n}$ is at least $1 / k$, the numbers of walks can then be identified, e.g., in terms of the limits of the successive derivatives $^{3}$ as $z>0$ goes to 0 :

$$
q(i, j ; n)=\lim _{\substack{z \rightarrow 0 \\(z>0)}} \frac{1}{n !}\left[\sum_{n \geq 0} q(i, j ; n) z^{n}\right]^{(n)} .
$$

Other implications of Theorems 1, 2 and 3 are presented in Section 7. Let us however make two remarks already.

Remark 1. Theorem 1 can be extended without difficulty to the case of the generating function counting the numbers of paths confined to the quadrant $\mathbb{Z}_{+}^{2}$, having length $k$, starting at $\left(i_{0}, j_{0}\right)$ and ending at $(i, j)$, for any initial state $\left(i_{0}, j_{0}\right)$.

Remark 2. A priori, the expression of $Q(x, 0 ; z)$ given in Theorem 1 is valid and holomorphic for $x$ inside of some curve (see Subsection 3.3) containing the branch points $x_{1}(z)$ and $x_{2}(z){ }^{4}$ This expression actually admits an analytic continuation onto $\mathbb{C} \backslash$ $\left[x_{3}(z), x_{4}(z)\right]$ : the arguments we gave for proving [17, Theorem 6] for Gessel's walk can indeed be applied. A similar remark holds for $Q(0, y ; z)$.

\footnotetext{
3 Throughout, for a function $v$ we denote by $[v]^{(n)}$ or by $\partial^{n} v$ its $n$th derivative.

4 Each term of the sum providing $c(x ; z) Q(x, 0 ; z)-c(0 ; z) Q(0,0 ; z)$ in Theorem 1 is clearly not holomorphic near $\left[x_{1}(z), x_{2}(z)\right]$, but an application of the residue theorem exactly as in $[18$, Section 4] would give an expression of the sum as a function clearly holomorphic near $\left[x_{1}(z), x_{2}(z)\right]$.
} 
Let us now turn to the five singular walks. Both functions $Q(x, 0 ; z)$ and $Q(0, y ; z)$ probably also admit integral representations (see [9, Part 6.4.1]), but here we prefer to give the following more elementary series representations. Below, by $f^{\circ p}$ we mean $f \circ \cdots \circ f$, with $p$ occurrences of $f$.

Theorem 4. Suppose that the walk is singular. The following series representation holds:

$Q(x, 0 ; z)=\frac{1}{z x^{2}} \sum_{p \geq 0} Y_{0} \circ\left(X_{0} \circ Y_{0}\right)^{\circ p}(x ; z)\left[\left(X_{0} \circ Y_{0}\right)^{\circ p}(x ; z)-\left(X_{0} \circ Y_{0}\right)^{\circ(p+1)}(x ; z)\right]$.

The function $Q(0, y ; z)$ is obtained from the equality above by exchanging the roles of $X_{0}$ and $Y_{0}$. Moreover, $Q(0,0 ; z)=0$, and the complete function $Q(x, y ; z)$ is obtained from (2).

The paper [21] gives a proof of Theorem 4 for the walks $\{N W, N E, S E\}$ and $\{N W, N, S E\}$, and suggests that this result should also hold for the other three singular walks. For the sake of completeness, in this article we prove Theorem 4 in all the five cases (see Subsection 3.4).

\section{Approach via boundary value problems}

\subsection{Structure of the remainder of the paper}

The approach that we are going to use depends on whether the walks under consideration are singular or not.

In order to prove Theorem 1 -this concerns all the non-singular walks, i.e., the 23 with a finite group as well as 51 of the 56 attached to an infinite group, see the tables in [6] —we here extend to three variables $x, y, z$ the profound analytic approach elaborated by Fayolle, Iasnogorodski and Malyshev in [9] for the stationary case, which correspond to two variables $x, y$. This is the aim of Subsection 3.3. To summarize:

Step 1. From the fundamental functional equation (2), we prove that $c(x ; z) Q(x, 0 ; z)$ and $\widetilde{c}(y ; z) Q(0, y ; z)$ satisfy certain boundary value problems of Riemann-Carleman type, i.e., with boundary conditions on curves closed in $\mathbb{C} \cup\{\infty\}$ and admitting non-empty interiors. These curves are studied in Lemma 2. The proof of this first step is performed in two stages. Firstly, we have to know the class of functions within which $c(x ; z) Q(x, 0 ; z)$ and $\widetilde{c}(y ; z) Q(0, y ; z)$ should be searched. This is the goal of Theorem 5 , which states that they admit nice holomorphic continuations in the whole interiors of the curves above. Its proof is postponed to Section 4 . Secondly, we have to obtain the precise boundary conditions on these curves; this is done in Subsection 3.3 (see (11)).

Step 2. Next we transform these problems into boundary value problems of RiemannHilbert type, i.e., with conditions on segments. This conversion is motivated by the fact that the latter problems are more usual and by far more treated in the literature (see $[9,19]$ and references therein). It is done in Subsection 3.3, by using conformal gluing functions in the sense of Definition 1. 
Step 3. Finally we solve these new problems and we deduce an explicit integral representation of (1). This will conclude Subsection 3.3.

Subsection 3.3 gives the complete proof of Theorem 1. As a consequence regarding the functions $c(x ; z) Q(x, 0 ; z)-c(0 ; z) Q(0,0 ; z)$ and $\widetilde{c}(y ; z) Q(0, y ; z)-\widetilde{c}(0 ; z) Q(0,0 ; z)$, it remains to find explicitly suitable conformal gluing functions $w$ and $\widetilde{w}$. This is the topic of Sections 5 and 6. In Section 6 we make an in-depth study of these conformal gluing functions, as their analysis is just sketched out in [9]. There we state and prove Theorem 6, which gives their explicit expression for all non-singular walks. In Section 6 we also prove Theorem 2 on the nature of $w$ and $\widetilde{w}$, as well as Theorem 3 giving simplified expressions of $w$ and $\widetilde{w}$ in the finite group case. The analysis of these functions relies on a uniformization of the Riemann surface given by the zeros of the kernel, namely, $\left\{(x, y) \in \mathbb{C}^{2}: \sum_{(i, j) \in \mathcal{S}} x^{i} y^{j}-1 / z=0\right\}$. This work is carried out in the introductoryand crucial-Section 5 .

For the five singular walks, the curves associated with the boundary value problems above degenerate to a point and the previous arguments no longer work. However, starting from (2), it is easy to make explicit a series representation of the function (1) (see Subsection 3.4).

\subsection{The kernel and its roots}

This part is introductory to Subsections 3.3 and 3.4. It aims at examining the kernel (4) that appears in (2), and in particular at studying its roots. First of all, we notice that the kernel can alternatively be written as

$$
\begin{aligned}
x y z\left[\sum_{(i, j) \in \mathcal{S}} x^{i} y^{j}-1 / z\right] & =\widetilde{a}(y ; z) x^{2}+\widetilde{b}(y ; z) x+\widetilde{c}(y ; z) \\
& =a(x ; z) y^{2}+b(x ; z) y+c(x ; z),
\end{aligned}
$$

where $\widetilde{c}(y ; z)$ and $c(x ; z)$ are defined in $(3)$, and where

$$
\begin{aligned}
& \widetilde{a}(y ; z)=z y \sum_{(+1, j) \in \mathcal{S}} y^{j}, \quad \tilde{b}(y ; z)=-1+z y \sum_{(0, j) \in \mathcal{S}} y^{j}, \\
& a(x ; z)=z x \sum_{(i,+1) \in \mathcal{S}} x^{i}, \quad b(x ; z)=-1+z x \sum_{(i, 0) \in \mathcal{S}} x^{i} .
\end{aligned}
$$

We also define

$$
\widetilde{d}(y ; z)=\widetilde{b}(y ; z)^{2}-4 \widetilde{a}(y ; z) \widetilde{c}(y ; z), \quad d(x ; z)=b(x ; z)^{2}-4 a(x ; z) c(x ; z) .
$$

- If the walk is non-singular, then for any $z \in] 0,1 / k[$, the polynomial $\tilde{d}$ (resp. $d$ ) has three or four roots, which we call $y_{k}(z)$ (resp. $\left.x_{k}(z)\right)$. As shown in [9, Part 2.3], they are such that $\left|y_{1}(z)\right|<y_{2}(z)<1<y_{3}(z)<\left|y_{4}(z)\right|$ (resp. $\left|x_{1}(z)\right|<x_{2}(z)<1<$ $\left.x_{3}(z)<\left|x_{4}(z)\right|\right)$, with $y_{4}(z)=\infty\left(\right.$ resp. $\left.x_{4}(z)=\infty\right)$ if $\tilde{d}($ resp. $d)$ has order three.

- If the walk is singular, the roots above then become $y_{1}(z)=y_{2}(z)=0<1<$ $y_{3}(z)<\left|y_{4}(z)\right|\left(\right.$ resp. $\left.x_{1}(z)=x_{2}(z)=0<1<x_{3}(z)<\left|x_{4}(z)\right|\right)$ (see [9, Part 6.1]). 
The behavior of the branch points $y_{k}(z)$ and $x_{k}(z)$ is not so simple for $\left.z \notin\right] 0,1 / k[$, and for this reason we suppose from now on that $z$ is fixed in $] 0,1 / k[$.

Now we notice that the kernel (4) vanishes if and only if $[\widetilde{b}(y ; z)+2 \widetilde{a}(y ; z) x]^{2}=$ $\widetilde{d}(y ; z)$ or $[b(x ; z)+2 a(x ; z) y]^{2}=d(x ; z)$. Consequently [15], the algebraic functions $X(y ; z)$ and $Y(x ; z)$ defined by

$$
\sum_{(i, j) \in \mathcal{S}} X(y ; z)^{i} y^{j}-1 / z=0 \text { and } \sum_{(i, j) \in \mathcal{S}} x^{i} Y(x ; z)^{j}-1 / z=0
$$

have two branches, meromorphic on the sets $\mathbb{C} \backslash\left(\left[y_{1}(z), y_{2}(z)\right] \cup\left[y_{3}(z), y_{4}(z)\right]\right)$ and $\mathbb{C} \backslash\left(\left[x_{1}(z), x_{2}(z)\right] \cup\left[x_{3}(z), x_{4}(z)\right]\right)\left(\right.$ resp. $\mathbb{C} \backslash\left[y_{3}(z), y_{4}(z)\right]$ and $\left.\mathbb{C} \backslash\left[x_{3}(z), x_{4}(z)\right]\right)$ in the non-degenerate (resp. degenerate) case.

We fix the notations of the two branches of the algebraic functions $X(y ; z)$ and $Y(x ; z)$ by setting

$$
X_{0}(y ; z)=\frac{-\widetilde{b}(y ; z)+\widetilde{d}(y ; z)^{1 / 2}}{2 \widetilde{a}(y ; z)}, \quad X_{1}(y ; z)=\frac{-\widetilde{b}(y ; z)-\widetilde{d}(y ; z)^{1 / 2}}{2 \widetilde{a}(y ; z)},
$$

as well as

$$
Y_{0}(x ; z)=\frac{-b(x ; z)+d(x ; z)^{1 / 2}}{2 a(x ; z)}, \quad Y_{1}(x ; z)=\frac{-b(x ; z)-d(x ; z)^{1 / 2}}{2 a(x ; z)} .
$$

The following straightforward result holds (see [9, Part 5.3]).

Lemma 1. For all $y \in \mathbb{C}$, we have $\left|X_{0}(y ; z)\right| \leq\left|X_{1}(y ; z)\right|$. Likewise, for all $x \in \mathbb{C}$, we have $\left|Y_{0}(x ; z)\right| \leq\left|Y_{1}(x ; z)\right|$.

\subsection{Non-singular walks: proof of Theorem 1}

In this subsection we show Theorem 1, dealing with all 74 non-singular walks. According to Subsection 3.1, we split the proof into three main steps.

Step 1. We prove that both functions $c(x ; z) Q(x, 0 ; z)$ and $\widetilde{c}(y ; z) Q(0, y ; z)$ satisfy a certain boundary value problem of Riemann-Carleman type, with boundary conditions on the curves

$$
X\left(\left[y_{1}(z), y_{2}(z)\right] ; z\right), \quad Y\left(\left[x_{1}(z), x_{2}(z)\right] ; z\right),
$$

respectively. Examples of the latter are represented in Figure 3. In the general case, they have the following properties (see [9, Part 5.3]).

Lemma 2. Let $X\left(\left[y_{1}(z), y_{2}(z)\right] ; z\right)$ and $Y\left(\left[x_{1}(z), x_{2}(z)\right] ; z\right)$.

- These two curves are symmetrical with respect to the real axis.

- They are connected and closed in $\mathbb{C} \cup\{\infty\}$.

- They split the plane into two connected components; let $\mathscr{G} X\left(\left[y_{1}(z), y_{2}(z)\right] ; z\right)$ and $\mathscr{G} Y\left(\left[x_{1}(z), x_{2}(z)\right] ; z\right)$ denote the ones of $x_{1}(z)$ and $y_{1}(z)$, respectively. They are such that $\mathscr{G} X\left(\left[y_{1}(z), y_{2}(z)\right] ; z\right) \subset \mathbb{C} \backslash\left[x_{3}(z), x_{4}(z)\right]$ and $\mathscr{G} Y\left(\left[x_{1}(z), x_{2}(z)\right] ; z\right) \subset$ $\mathbb{C} \backslash\left[y_{3}(z), y_{4}(z)\right]$. 

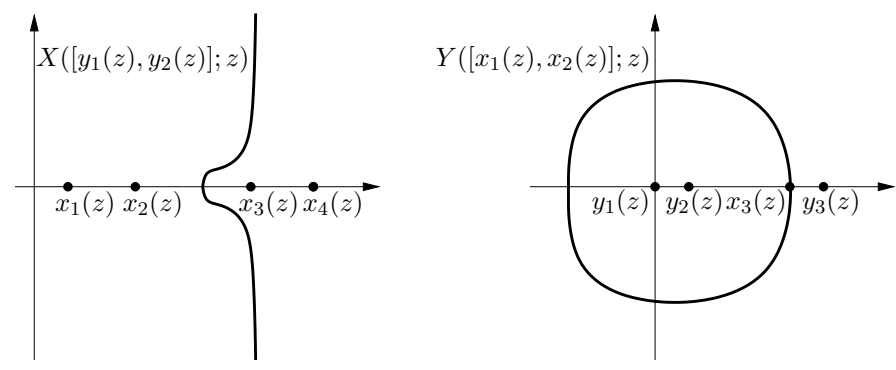

Fig. 3. The curves $X\left(\left[y_{1}(z), y_{2}(z)\right] ; z\right)$ and $Y\left(\left[x_{1}(z), x_{2}(z)\right] ; z\right)$ for Gessel's walk.

As illustrated by the example of Gessel's walk (see again Figure 3), these curves are not always included in the unit disc, so that the functions $c(x ; z) Q(x, 0 ; z)$ and $\widetilde{c}(y ; z) Q(0, y ; z)$ a priori need not be defined on them. For this reason, we first have to continue the generating functions up to these curves: this is exactly the object of the following result, the proof of which is the subject of Section 4.

Theorem 5. The functions $c(x ; z) Q(x, 0 ; z)$ and $\widetilde{c}(y ; z) Q(0, y ; z)$ can be holomorphically continued from the open unit disc $\mathscr{D}$ to $\mathscr{G} X\left(\left[y_{1}(z), y_{2}(z)\right] ; z\right) \cup \mathscr{D}$ and $\mathscr{G} Y\left(\left[x_{1}(z), x_{2}(z)\right] ; z\right) \cup \mathscr{D}$, respectively.

Now, exactly as in [17, Section 1], we obtain:

$\forall t \in X\left(\left[y_{1}(z), y_{2}(z)\right] ; z\right), \quad c(t ; z) Q(t, 0 ; z)-c(\bar{t} ; z) Q(\bar{t}, 0 ; z)=t Y_{0}(t ; z)-\bar{t} Y_{0}(\bar{t} ; z)$,

$\forall t \in Y\left(\left[x_{1}(z), x_{2}(z)\right] ; z\right), \quad \widetilde{c}(t ; z) Q(0, t ; z)-\widetilde{c}(\bar{t} ; z) Q(0, \bar{t} ; z)=X_{0}(t ; z) t-X_{0}(\bar{t} ; z) \bar{t}$.

Together with Theorem 5, we thus conclude that $c(x ; z) Q(x, 0 ; z)$ and $\widetilde{c}(y ; z) Q(0, y ; z)$ are to be looked for among the functions holomorphic in $\mathscr{G} X\left(\left[y_{1}(z), y_{2}(z)\right] ; z\right)$ and $\mathscr{G} Y\left(\left[x_{1}(z), x_{2}(z)\right] ; z\right)$ and satisfying the conditions $(11)$ on the boundary of these sets.

Step 2. A standard [9, 19] way to solve these boundary value problems consists in converting them into problems with boundary conditions on segments. This transformation is performed by the use of conformal gluing functions, defined below.

Definition 1. Let $\mathscr{C} \subset \mathbb{C} \cup\{\infty\}$ be an open and simply connected set, symmetrical with respect to the real axis, and not equal to $\emptyset, \mathbb{C}$ and $\mathbb{C} \cup\{\infty\}$. A function $w$ is said to be a conformal gluing function (CGF) for the set $\mathscr{C}$ if

- $w$ is meromorphic in $\mathscr{C}$;

- $w$ establishes a conformal mapping of $\mathscr{C}$ onto the complex plane cut along a segment;

- For all $t$ in the boundary of $\mathscr{C}, w(t)=w(\bar{t})$.

Remark 3. It is worth noting that the existence (but without any explicit expression) of a CGF for a generic set $\mathscr{C}$ is ensured by general results on conformal gluing [19, Chapter 2].

Let $w(t ; z)$ be a CGF for the set $\mathscr{G} X\left(\left[y_{1}(z), y_{2}(z)\right] ; z\right)$ and let $U_{z}$ denote the real segment $w\left(X\left(\left[y_{1}(z), y_{2}(z)\right] ; z\right) ; z\right)$. We also define $v(u ; z)$ as the inverse function of $w(t ; z)$. 
It is meromorphic on $\mathbb{C} \backslash U_{z}$ (see Definition 1). Finally, for real values of $u$ we write $v^{+}(u ; z)$ (resp. $\left.v^{-}(u ; z)\right)$ for the limit of $v(s ; z)$ as $s \rightarrow u$ from the upper (resp. lower) half-plane. We notice that for $u \in U_{z}, v^{+}(u ; z)$ and $v^{-}(u ; z)$ are different and complex conjugate to one another.

With the notations above, the boundary value problem of the first step becomes that of finding a function $c(v(u ; z) ; z) Q(v(u ; z), 0 ; z)$ holomorphic in $\mathbb{C} \backslash U_{z}$, bounded near the ends of $U_{z}$, and such that, for $u \in U_{z}$,

$$
\begin{aligned}
c\left(v^{+}(u ; z) ; z\right) Q\left(v^{+}(u ; z), 0 ; z\right)-c\left(v^{-}(u ; z) ; z\right) Q\left(v^{-}(u ; z), 0 ; z\right) & \\
& =v^{+}(u ; z) Y_{0}\left(v^{+}(u ; z) ; z\right)-v^{-}(u ; z) Y_{0}\left(v^{-}(u ; z) ; z\right) .
\end{aligned}
$$

Step 3. This problem is standard [9, 19] and can be immediately resolved. It yields, up to an additive function of $z$,

$$
\begin{aligned}
& c(v(u ; z) ; z) Q(v(u ; z), 0 ; z) \\
& \quad=\frac{1}{2 \pi \imath} \int_{U_{z}} \frac{v^{+}(s ; z) Y_{0}\left(v^{+}(s ; z) ; z\right)-v^{-}(s ; z) Y_{0}\left(v^{-}(s ; z) ; z\right)}{s-u} d s .
\end{aligned}
$$

The change of variable $s=w(t ; z)$ then gives, up to an additive function of $z$,

$$
c(x ; z) Q(x, 0 ; z)=\frac{1}{2 \pi \imath} \int_{X\left(\left[y_{1}(z), y_{2}(z)\right] ; z\right)} t Y_{0}(t) \frac{\partial_{t} w(t ; z)}{w(t ; z)-w(x ; z)} d t .
$$

We note that for the difference $c(x ; z) Q(x, 0 ; z)-c(0 ; z) Q(0,0 ; z)$ considered in Theorem 1, it is not worth knowing the additive function of $z$ appearing in (12). The residue theorem applied exactly as in [17, Section 4] then transforms the integral on a curve (12) into the integral on a segment written in Theorem 1, and the proof of the formula for $c(x ; z) Q(x, 0 ; z)-c(0 ; z) Q(0,0 ; z)$ is completed.

The expression of $\widetilde{c}(y ; z) Q(0, y ; z)-\widetilde{c}(0 ; z) Q(0,0 ; z)$ is derived similarly. Suppose now that SW $\notin \mathcal{S}$, or equivalently that $\widetilde{c}(0 ; z)=0$. The last formula then yields an expression of $\widetilde{c}(y ; z) Q(0, y ; z)$, whence of $Q(0, y ; z)$ by division, and finally of $Q(0,0 ; z)$ by substitution. If now $\mathrm{SW} \in \mathcal{S}$, evaluating the functional equation (2) at any $\left(x_{0}, y_{0}, z_{0}\right) \in$ $\{|x| \leq 1,|y| \leq 1,|z|<1 / k\}$ at which the kernel (4) vanishes immediately provides the expression of $Q(0,0 ; z)$ stated in Theorem 1 . As for $Q(x, y ; z)$, it is then sufficient to use the functional equation.

\subsection{Singular walks: proof of Theorem 4}

Theorem 4 is shown in [21] for both step sets $\mathcal{S}=\{N W, N E, S E\}$ and $\{N W, N, S E\}$. In this subsection we explain how to obtain it for all five singular walks. Along the same lines as in [9, Part 6.4], we obtain from (2) the identity

$$
\begin{aligned}
c\left(X_{0} \circ Y_{0}(t ; z) ; z\right) Q\left(X_{0} \circ Y_{0}(t ; z), 0 ; z\right) & -c(t ; z) Q(t, 0 ; z) \\
& =X_{0} \circ Y_{0}(t ; z) Y_{0}(t ; z)-t Y_{0}(t ; z) .
\end{aligned}
$$


Applying (13) for $t=\left(X_{0} \circ Y_{0}\right)^{\circ p}(x ; z)$ and summing with respect to $p \in\{0, \ldots, q\}$ formally gives, with the same notations as in the statement of Theorem 4 ,

$$
\begin{aligned}
& c\left(\left(X_{0} \circ Y_{0}\right)^{\circ(q+1)}(x ; z) ; z\right) Q\left(\left(X_{0} \circ Y_{0}\right)^{\circ(q+1)}(x ; z), 0 ; z\right)-c(x ; z) Q(x, 0 ; z) \\
& \quad=\sum_{p=0}^{q} Y_{0} \circ\left(X_{0} \circ Y_{0}\right)^{\circ p}(x ; z)\left[\left(X_{0} \circ Y_{0}\right)^{\circ p}(x ; z)-\left(X_{0} \circ Y_{0}\right)^{\circ(p+1)}(x ; z)\right] .
\end{aligned}
$$

Using [9, Part 6.4.2], i.e., the fact that (at least) for $|x|<1$ and $|z|<1 / k$, we have $\left|X_{0} \circ Y_{0}(x ; z)\right|<x$, gives that the series above is convergent as $q \rightarrow \infty$. This also implies that the left-hand side goes to $c(0 ; z) Q(0,0 ; z)-c(x ; z) Q(x, 0 ; z)=-z x^{2} Q(x, 0 ; z)$ as $q \rightarrow \infty$, since for all five singular walks $c(x ; z)=z x^{2}$ (see (3)). Theorem 4 follows.

\section{Holomorphic continuation of the generating functions}

This part aims at proving Theorem 5. In other words, we must show that the generating functions $c(x) Q(x, 0)^{5}$ and $\widetilde{c}(y) Q(0, y)$, already known to be holomorphic in the open unit disc $\mathscr{D}$, can be holomorphically continued up to $\mathscr{G} X\left(\left[y_{1}, y_{2}\right]\right) \cup \mathscr{D}$ and $\mathscr{G} Y\left(\left[x_{1}, x_{2}\right]\right) \cup \mathscr{D}$, respectively. First of all, note that the location of the sets $\mathscr{G} X\left(\left[y_{1}, y_{2}\right]\right)$ and $\mathscr{G} Y\left(\left[x_{1}, x_{2}\right]\right)$ depends strongly on the step set $\mathcal{S}$. In particular, it may happen that they are included in the unit disc-e.g., it is the case for the walks $\{N, S E, W\}$ and $\{N, E, S E, S, W, N W\}$, as will be further illustrated in Figure 4-and in that case Theorem 5 is obvious. On the other hand, there actually exist walks for which these sets do not lie inside the unit disc-for example, Gessel's walk (see Figure 3). The proof of Theorem 5 requires the following results.

Lemma 3. Assume that the walk is non-singular. The following properties hold:

(i) $Y_{0}(\{|x|=1\}) \subset\{|y|<1\}$;

(ii) $X_{0}: \mathscr{G} Y\left(\left[x_{1}, x_{2}\right]\right) \backslash\left[y_{1}, y_{2}\right] \rightarrow \mathscr{G} X\left(\left[y_{1}, y_{2}\right]\right) \backslash\left[x_{1}, x_{2}\right]$ and $Y_{0}: \mathscr{G} X\left(\left[y_{1}, y_{2}\right]\right) \backslash$ $\left[x_{1}, x_{2}\right] \rightarrow \mathscr{G} Y\left(\left[x_{1}, x_{2}\right]\right) \backslash\left[y_{1}, y_{2}\right]$ are conformal and inverse to one another;

(iii) $\left\{x \in \mathbb{C}:\left|Y_{0}(x)\right|<1\right\} \cap \mathscr{D}$ is non-empty;

(iv) $\mathscr{G} X\left(\left[y_{1}, y_{2}\right]\right) \cup \mathscr{D}$ is connected;

(v) $\mathscr{G} X\left(\left[y_{1}, y_{2}\right]\right) \cup \mathscr{D}$ is included in $\left\{x \in \mathbb{C}:\left|Y_{0}(x)\right|<1\right\} \cup \mathscr{D}$.

Proof. Let us recall that $Y_{0}$ is one of the two $y$-roots of the kernel (4), and that with $Y_{1}$ denoting the other one, we have $\left|Y_{0}\right| \leq\left|Y_{1}\right|$ (see Lemma 1). We are going to prove (i) first for $z=1 / k$, and we shall deduce from this the remaining cases $z \in] 0,1 / k[$.

- Assume that $z=1 / k$. If the kernel (4) vanishes at $(x, y)$ then $\sum_{(i, j) \in \mathcal{S}}(1 / k) x^{i} y^{j}$ $=1$. Since $\sum_{(i, j) \in \mathcal{S}} 1 / k=1$, we can apply [9, Lemma 2.3.4], and in this way we find that $Y_{0}(\{|x|=1\}) \subset\{|y| \leq 1\}$.

\footnotetext{
5 For the sake of conciseness we will, from now on, drop the dependence of the different quantities on $z \in] 0,1 / k[$. Moreover, it is implied that any statement in what follows begins with "for any $z \in] 0,1 / k["$.
} 
- Suppose that $z \in] 0,1 / k[$. In that case, the kernel cannot vanish at $(x, y)$ with $|x|=$ $|y|=1$ : indeed, for $|x|=|y|=1$ we clearly have $\left|\sum_{(i, j) \in \mathcal{S}} x^{i} y^{j}\right| \leq \sum_{(i, j) \in \mathcal{S}} 1=$ $k<1 / z$. As a consequence, $Y_{0}(\{|x|=1\}) \cap\{|y|=1\}$ is empty. By connectedness, this implies that either $Y_{0}(\{|x|=1\}) \subset\{|y|<1\}$ or $Y_{0}(\{|x|=1\}) \subset\{|y|>1\}$. But once again by [9, Lemma 2.3.4], for $z=1 / k$ we obtain $Y_{0}(\{|x|=1\}) \cap\{|y|<1\} \neq \emptyset$, so that by continuity we deduce that $Y_{0}(\{|x|=1\}) \subset\{|y|<1\}$ for all $\left.z \in\right] 0,1 / k[$.

Item (ii) is proved in [9, Part 5.3] for $z=1 / k$; the proof for other values of $z$ is similar and we therefore choose to omit it. Note now that (iii) is a straightforward consequence of (i). Item (iv) is also clear: both sets $\mathscr{G} X\left(\left[y_{1}, y_{2}\right]\right)$ and $\mathscr{D}$ are connected and their intersection is non-empty, since $x_{1}$ belongs to both sets. For (v), it is enough to prove that $\left(\mathscr{G} X\left(\left[y_{1}, y_{2}\right]\right) \cup \mathscr{D}\right) \backslash \mathscr{D}$ is included in $\left\{x \in \mathbb{C}:\left|Y_{0}(x)\right|<1\right\}$. This will follow from an application of the maximum modulus principle (see, e.g., [15]) to the function $Y_{0}$ on $\left(\mathscr{G} X\left(\left[y_{1}, y_{2}\right]\right) \cup \mathscr{D}\right) \backslash \mathscr{D}$. First of all note that $Y_{0}$ is analytic on the latter domain, since thanks to Subsections 3.2 and 3.3 it is included in $\mathbb{C} \backslash\left(\left[x_{1}, x_{2}\right] \cup\left[x_{3}, x_{4}\right]\right)$. Next, we prove that $\left|Y_{0}\right|<1$ on the boundary of the set $\left(\mathscr{G} X\left(\left[y_{1}, y_{2}\right]\right) \cup \mathscr{D}\right) \backslash \mathscr{D}$, and for this it is sufficient to show that $\left|Y_{0}\right|<1$ on $\{|x|=1\} \cup X\left(\left[y_{1}, y_{2}\right]\right)$. But by (i) it is immediate that $\left|Y_{0}\right|<1$ on $\{|x|=1\}$, and by (ii) we get $Y_{0}\left(X\left(\left[y_{1}, y_{2}\right]\right)\right)=\left[y_{1}, y_{2}\right]$, a segment which is known to lie in the unit disc, thanks to Subsection 3.2. In this way, the maximum modulus principle directly entails that $\left|Y_{0}\right|<1$ on $\left(\mathscr{G} X\left(\left[y_{1}, y_{2}\right]\right) \cup \mathscr{D}\right) \backslash \mathscr{D}$.

Proof of Theorem 5. We are going to explain here the continuation procedure only for $c(x) Q(x, 0)$, since the case of $\widetilde{c}(y) Q(0, y)$ is similar.

Evaluating (2) at any $(x, y) \in\{|x| \leq 1,|y| \leq 1\}$ such that $\sum_{(i, j) \in \mathcal{S}} x^{i} y^{j}-1 / z=0$ leads to $c(x) Q(x, 0)+\widetilde{c}(y) Q(0, y)-z \delta Q(0,0)-x y=0$. Therefore, if $x \in\{x \in \mathbb{C}:$ $\left.\left|Y_{0}(x)\right|<1\right\} \cap \mathscr{D}$, we obtain

$$
c(x) Q(x, 0)+\widetilde{c}\left(Y_{0}(x)\right) Q\left(0, Y_{0}(x)\right)-z \delta Q(0,0)-x Y_{0}(x)=0 .
$$

Since $\left\{x \in \mathbb{C}:\left|Y_{0}(x)\right|<1\right\} \cap \mathscr{D}$ is non-empty (see Lemma 3(iii)), both functions $c(x) Q(x, 0)$ and $\widetilde{c}\left(Y_{0}(x)\right) Q\left(0, Y_{0}(x)\right)$ as well as the identity (14) can be extended to the connected component of $\left\{x \in \mathbb{C}:\left|Y_{0}(x)\right|<1\right\} \cup \mathscr{D}$ containing $\left\{x \in \mathbb{C}:\left|Y_{0}(x)\right|<1\right\} \cap \mathscr{D}$, by analytic continuation.

As a consequence, we now need to show that $\mathscr{G} X\left(\left[y_{1}, y_{2}\right]\right) \cup \mathscr{D}$ is connected and included in $\left\{x \in \mathbb{C}:\left|Y_{0}(x)\right|<1\right\} \cup \mathscr{D}$, since $\mathscr{G} X\left(\left[y_{1}, y_{2}\right]\right) \cup \mathscr{D}$ clearly has a non-empty intersection with $\left\{x \in \mathbb{C}:\left|Y_{0}(x)\right|<1\right\} \cap \mathscr{D}$. These facts are exactly the objects of Lemma 3(iv) and (v).

It thus remains to prove that this continuation of $c(x) Q(x, 0)$ is holomorphic on $\mathscr{G} X\left(\left[y_{1}, y_{2}\right]\right) \cup \mathscr{D}$.

- On $\mathscr{D}$ this is immediate, $c(x) Q(x, 0)$ being therein defined by its power series.

- On $\left(\mathscr{G} X\left(\left[y_{1}, y_{2}\right]\right) \cup \mathscr{D}\right) \backslash \mathscr{D}$, it follows from (14) that $c(x) Q(x, 0)$ may possibly have the same singularities as $Y_{0}$-namely, the branch cuts $\left[x_{1}, x_{2}\right],\left[x_{3}, x_{4}\right]$ - and is holomorphic elsewhere. But these segments do not belong to $\left(\mathscr{G} X\left(\left[y_{1}, y_{2}\right]\right) \cup \mathscr{D}\right) \backslash \mathscr{D}$ : by Subsection 3.2 we know that $\left[x_{1}, x_{2}\right]$ is included in $\mathscr{D}$, and from Lemma 2 we see that $\left[x_{3}, x_{4}\right]$ is exterior to $\mathscr{G} X\left(\left[y_{1}, y_{2}\right]\right)$. 
The continuation of $c(x) Q(x, 0)$ is thus holomorphic on $\mathscr{G} X\left(\left[y_{1}, y_{2}\right]\right) \cup \mathscr{D}$ and Theorem 5 is proved.

Let us note that in [17], we also introduced a procedure of continuation of $c(x) Q(x, 0)$ and $\widetilde{c}(y) Q(0, y)$. We have chosen to present here another way: it is weaker since Theorem 5 yields a continuation of the generating functions up to $\mathscr{G} X\left(\left[y_{1}, y_{2}\right]\right) \cup \mathscr{D}$ and $\mathscr{G} Y\left(\left[x_{1}, x_{2}\right]\right) \cup \mathscr{D}$ and not to $\mathbb{C} \backslash\left[x_{3}, x_{4}\right]$ and $\mathbb{C} \backslash\left[y_{3}, y_{4}\right]$ as in [17]; it is, however, more elementary, because this continuation is performed directly on the complex plane, rather than on a uniformization of the set given by the zeros of the kernel (4) as in [17].

\section{Uniformization}

This part serves to introduce notions that are crucial to Section 6. It amounts to studying closely the set of zeros of the kernel (4),

$$
\mathscr{K}=\left\{(x, y) \in \mathbb{C}^{2}: \sum_{(i, j) \in \mathcal{S}} x^{i} y^{j}-1 / z=0\right\} .
$$

Proposition 2. For any non-singular walk, $\mathscr{K}$ is a Riemann surface of genus one.

Proof. Subsection 3.2 shows that $\sum_{(i, j) \in \mathcal{S}} x^{i} y^{j}-1 / z=0$ if and only if $[b(x)+2 a(x) y]^{2}$ $=d(x)$. But the Riemann surface of the square root of a third- or fourth-degree polynomial with distinct roots has genus one (see, e.g., [15]). Therefore the genus of $\mathscr{K}$ is also one.

Remark 4. Note that Proposition 2 cannot be extended to the singular walks. Indeed, it follows from Subsection 3.2 that for these walks, the polynomial $d$ has a double root at 0 and two simple roots at $x_{3}$ and $x_{4}$, and it is well known (see [15]) that the Riemann surface of the square root of such a polynomial has genus zero. We also notice that Proposition 2, implicitly stated for $z \in] 0,1 / k$, cannot be extended to $z=0$ or $z=1 / k$ in the general case. Indeed, for $z=0$ we have $d(x)=x^{2}$ and the Riemann surface of the square root of this polynomial is a disjoint union of two spheres (see [15]). For $z=1 / k$, it may happen that the genus of $\mathscr{K}$ is still one, but it may also happen that it becomes zero. In fact, [9, Parts 2.3 and 6.1] entails that it equals zero if and only if $\sum_{(i, j) \in \mathcal{S}} i=0$ and $\sum_{(i, j) \in \mathcal{S}} j=0$.

From Proposition 2, it is immediate that $\mathscr{K}$ is isomorphic to a certain torus $\mathbb{C} / \Omega$. A suitable lattice $\Omega$-in fact the only possible lattice, up to a homothetic transformationis made explicit in [9, Parts 3.1 and 3.3], namely, $\Omega=\omega_{1} \mathbb{Z}+\omega_{2} \mathbb{Z}$ with

$$
\omega_{1}=\imath \int_{x_{1}}^{x_{2}} \frac{d x}{[-d(x)]^{1 / 2}}, \quad \omega_{2}=\int_{x_{2}}^{x_{3}} \frac{d x}{[d(x)]^{1 / 2}} .
$$

Using the same arguments as in [9, Part 3.3], we immediately obtain in addition the uniformization

$$
\mathscr{K}=\{(x(\omega), y(\omega)): \omega \in \mathbb{C} / \Omega\},
$$

with

$$
x(\omega)=F\left(\wp(\omega), \wp^{\prime}(\omega)\right), \quad y(\omega)=G\left(\wp(\omega), \wp^{\prime}(\omega)\right),
$$


where $F\left(p, p^{\prime}\right)=x_{4}+d^{\prime}\left(x_{4}\right) /\left[p-d^{\prime \prime}\left(x_{4}\right) / 6\right]$ and $G\left(p, p^{\prime}\right)=\left[-b\left(F\left(p, p^{\prime}\right)\right)+\right.$ $\left.d^{\prime}\left(x_{4}\right) p^{\prime} /\left(2\left[p-d^{\prime \prime}\left(x_{4}\right) / 6\right]^{2}\right)\right] /\left[2 a\left(F\left(p, p^{\prime}\right)\right)\right]$ if $x_{4} \neq \infty$, while $F\left(p, p^{\prime}\right)=$ $\left[6 p-d^{\prime \prime}(0)\right] / d^{\prime \prime \prime}(0)$ and $G\left(p, p^{\prime}\right)=\left[-b\left(F\left(p, p^{\prime}\right)\right)-3 p^{\prime} / d^{\prime \prime \prime}(0)\right] /\left[2 a\left(F\left(p, p^{\prime}\right)\right)\right]$ if $x_{4}=\infty$, and where $\wp$ denotes the Weierstrass elliptic function with periods $\omega_{1}, \omega_{2}$. By definition (see [15, 24]), $\wp$ is equal to

$$
\wp(\omega)=\frac{1}{\omega^{2}}+\sum_{\left(p_{1}, p_{2}\right) \in \mathbb{Z}^{2} \backslash(0,0)}\left[\frac{1}{\left(\omega-p_{1} \omega_{1}-p_{2} \omega_{2}\right)^{2}}-\frac{1}{\left(p_{1} \omega_{1}+p_{2} \omega_{2}\right)^{2}}\right],
$$

and it is well known that it satisfies the differential equation

$$
\wp^{\prime}(\omega)^{2}=4\left[\wp(\omega)-\wp\left(\omega_{1} / 2\right)\right]\left[\wp(\omega)-\wp\left(\left[\omega_{1}+\omega_{2}\right] / 2\right)\right]\left[\wp(\omega)-\wp\left(\omega_{2} / 2\right)\right] .
$$

The main motivation for introducing a uniformization of $\mathscr{K}$ is to render the roles of $x$ and $y$ more symmetric. This is why we now go one step further, and we look for the location on $\mathbb{C}$, or equivalently on the fundamental parallelogram $\left[0, \omega_{2}\left[\times\left[0, \omega_{1} /{ }_{l}[\right.\right.\right.$, of the inverse images through the uniformization (16) of the important cycles that are the branch cuts $\left[x_{1}, x_{2}\right],\left[x_{3}, x_{4}\right],\left[y_{1}, y_{2}\right]$ and $\left[y_{3}, y_{4}\right]$. For the first two, we introduce

$$
f(t)= \begin{cases}d^{\prime \prime}\left(x_{4}\right) / 6+d^{\prime}\left(x_{4}\right) /\left[t-x_{4}\right] & \text { if } x_{4} \neq \infty, \\ d^{\prime \prime}(0) / 6+d^{\prime \prime \prime}(0) t / 6 & \text { if } x_{4}=\infty .\end{cases}
$$

It is such that $x(\omega)=f^{-1}(\wp(\omega))$ (see (16)). Further, it follows from the construction that $\wp\left(\omega_{1} / 2\right)=f\left(x_{3}\right), \wp\left(\left[\omega_{1}+\omega_{2}\right] / 2\right)=f\left(x_{2}\right)$ and $\wp\left(\omega_{1} / 2\right)=f\left(x_{1}\right)$; for a proof, see [9, Part 3.3]. As for $\left[y_{1}, y_{2}\right]$ and $\left[y_{3}, y_{4}\right]$, we need to introduce a new period, namely,

$$
\omega_{3}=\int_{X\left(y_{1}\right)}^{x_{1}} \frac{d x}{[d(x)]^{1 / 2}} .
$$

In [9, Part 3.3], the following is shown.

Lemma 4. The period $\omega_{3}$ lies in $] 0, \omega_{2}[$.

By using the same analysis of [9, Part 5.5], we obtain the following pleasing result.

Proposition 3. We have

$$
\begin{array}{ll}
x^{-1}\left(\left[x_{1}, x_{2}\right]\right)=\left[0, \omega_{1}\left[+\omega_{2} / 2,\right.\right. & x^{-1}\left(\left[x_{3}, x_{4}\right]\right)=\left[0, \omega_{1}[,\right. \\
y^{-1}\left(\left[y_{1}, y_{2}\right]\right)=\left[0, \omega_{1}\left[+\left(\omega_{2}+\omega_{3}\right) / 2,\right.\right. & y^{-1}\left(\left[y_{3}, y_{4}\right]\right)=\left[0, \omega_{1}\left[+\omega_{3} / 2 .\right.\right.
\end{array}
$$

In our opinion, the latter result justifies the introduction of the uniformization: indeed, on the fundamental parallelogram, the quartic curves $X\left(\left[y_{1}, y_{2}\right]\right)$ and $Y\left(\left[x_{1}, x_{2}\right]\right)$ just become segments! Proposition 3 in particular asserts that $\omega_{3}$ naturally appears when locating the branch cuts on the uniformization space. In fact, this period also plays a crucial role with respect to the group $W=\langle\Psi, \Phi\rangle$ defined in (5). Indeed, the two birational transformations $\Psi$ and $\Phi$ of $\mathbb{C}^{2}$ can a fortiori be understood as automorphisms of $\mathscr{K}$, and we 
recall $[9$, Part 3.1] that thanks to (16), these automorphisms of $\mathscr{K}$ become on $\mathbb{C} / \Omega$ the automorphisms $\psi$ and $\phi$ with the following expressions:

$$
\psi(\omega)=-\omega, \quad \phi(\omega)=-\omega+\omega_{3} .
$$

They satisfy $\psi \circ \psi=\phi \circ \phi=\mathrm{id}, x \circ \psi=x, y \circ \psi=[c(x) / a(x)] / y, x \circ \phi=[\widetilde{c}(y) / \widetilde{a}(y)] / x$ and $y \circ \phi=y$.

Our goal now is to find a characterization of the finiteness of the group in terms of $\omega_{3}$. We shall obtain it in Proposition 5. The latter requires us the preliminary result hereunder.

Lemma 5. We have $\omega_{3}<\omega_{2} / 2$ (resp. $\left.\omega_{3}=\omega_{2} / 2, \omega_{3}>\omega_{2} / 2\right)$ if and only if the covariance (6) of the walk is negative (resp. zero, positive).

Proof. We just give the sketch of the proof; for the details, we refer to [18, Section 4]. The first step consists in noticing that $\omega_{3}=\omega_{2} / 2$ if and only if the covariance equals zero, which follows by showing that both assertions are equivalent to having a group of order 4 . This essentially implies that it suffices to find one walk for which we simultaneously have $\omega_{3}<\omega_{2} / 2$ (resp. $\omega_{3}<\omega_{2} / 2$ ) and a negative (resp. positive) covariance. This verification is performed in [18] for the walk $\{E, S, N W\}$ (resp. $\{N E, S, W\}$ ).

It could appear surprising to introduce the covariance here. As we will see throughout, its sign actually strongly influences the behavior of many quantities (see Theorem 2, itself summarized in Figure 6). If the group $W=\langle\Psi, \Phi\rangle$ is finite, we can give the following important specifications to Lemma 5.

Proposition 4. For any $k \geq 2$, the group $W=\langle\Psi, \Phi\rangle$ has order $2 k$ if and only if there exists an integer $q \in\{1, \ldots, k-1\}$, independent of $z$ and having no common divisors with $k$, such that for all $z \in] 0,1 / k\left[, \omega_{3}=(q / k) \omega_{2}\right.$.

Proof. We first prove the converse direction and for this purpose, we reintroduce the variable $z$ in the notations.

Assume first that for some $z \in] 0,1 / k\left[\right.$, one has $\omega_{3} / \omega_{2}=q / k$, where the integers $q$ and $k$ have no common divisors. This means that $\langle\psi, \phi\rangle$ is a finite group of order $2 k$ (see (20)), which is equivalent to the fact that $\langle\Psi, \Phi\rangle$ has order $2 k$ on $\mathscr{K}$-but a priori not on $\mathbb{C}^{2}$. This implies that for any $x \in \mathbb{C}$, we have $(\Phi \circ \Psi)^{\circ k}\left(x, Y_{0}(x ; z)\right)=\left(x, Y_{0}(x ; z)\right)$ and $(\Phi \circ \Psi)^{\circ k}\left(x, Y_{1}(x ; z)\right)=\left(x, Y_{1}(x ; z)\right)$ hold.

Suppose now that for any $z \in] 0,1 / k\left[\right.$, the quantity $\omega_{3} / \omega_{2}$ is this same rational number $q / k$. In particular for any fixed $x \in \mathbb{C}$ and all $z \in] 0,1 / k\left[,(\Phi \circ \Psi)^{\circ k}\left(x, Y_{0}(x ; z)\right)=\right.$ $\left(x, Y_{0}(x ; z)\right)$. In other words, for any fixed $x \in \mathbb{C}$ and any $y \in\left\{Y_{0}(x ; z): z \in\right] 0,1 / k[\}$, $(\Phi \circ \Psi)^{\circ k}(x, y)=(x, y)$. Since the set $\left\{Y_{0}(x ; z): z \in\right] 0,1 / k[\}$ is not isolated, by analytic continuation we find that for all $x \in \mathbb{C}$ and $y \in \mathbb{C},(\Phi \circ \Psi)^{\circ k}(x, y)=(x, y)$, so that $\langle\Psi, \Phi\rangle$ is finite as a group of birational transformations of $\mathbb{C}^{2}$, of order less than or equal to $2 k$.

The group $\langle\Psi, \Phi\rangle$ has order exactly $2 k$ because $\langle\psi, \phi\rangle$ has order $2 k$ and thus we can find some elements $(x, y)$ of order exactly $2 k$. This entails the converse implication of Proposition 4. 
Suppose now that $W=\langle\Psi, \Phi\rangle$ has order $2 k$. The group $\langle\psi, \phi\rangle$ is a fortiori finite, of order $2 r(z) \leq 2 k$, which means that $\inf \left\{p>0:(\phi \circ \psi)^{\circ p}=\mathrm{id}\right\}=r(z)$. With (20) this immediately implies that $r(z) \omega_{3}$ is a point of the lattice $\omega_{1} \mathbb{Z}+\omega_{2} \mathbb{Z}$, in contrast to $p \omega_{3}$ for $p \in\{1, \ldots, r(z)-1\}$. But by Lemma 4 we have $\left.\omega_{3} \in\right] 0, \omega_{2}\left[\right.$, so that $r(z) \omega_{3}=q(z) \omega_{2}$, where $q(z) \in\{1, \ldots, r(z)-1\}$ has no common divisors with $r(z)$.

Moreover, thanks to (15) and (19) we know that $\omega_{3} / \omega_{2}=q(z) / r(z)$ is a holomorphic function of $z$; taking rational values it has to be constant, say $\omega_{3} / \omega_{2}=q / r$. Finally, if $r$ were strictly smaller than $k$, then the first part of the proof would imply that $W=\langle\Psi, \Phi\rangle$ has also order $2 r$, and not $2 k$ as assumed, so that $r=k$ and Proposition 4 is proved.

In particular, since it is proved in [6] that the group $W$ can only have order $4,6,8$ or $\infty$, Lemma 5 and Proposition 4 immediately lead to the following result.

Proposition 5. The walk is associated with a group $W=\langle\Psi, \Phi\rangle$ of order 4 if and only if for all $z \in] 0,1 / k\left[, \omega_{3}=\omega_{2} / 2\right.$. For $k \in\{3,4\}$, the walk has a group of order $2 k$ and a negative (resp. positive) covariance (6) if and only if for all $z \in] 0,1 / k\left[, \omega_{3}=\omega_{2} / k\right.$ (resp. $\left.\omega_{3}=\omega_{2}-\omega_{2} / k\right)$ ).

The location of the inverse images of $\left[x_{1}, x_{2}\right],\left[x_{3}, x_{4}\right],\left[y_{1}, y_{2}\right]$ and $\left[y_{3}, y_{4}\right]$ through (16) being known (see Proposition 3), we give in Figure 4 two examples of the parallelogram $\left[0, \omega_{2}\left[\times\left[0, \omega_{1} / l[\right.\right.\right.$ with its important cycles-in addition to the one corresponding to Gessel's walk, which can be found in [17, Figure 5].

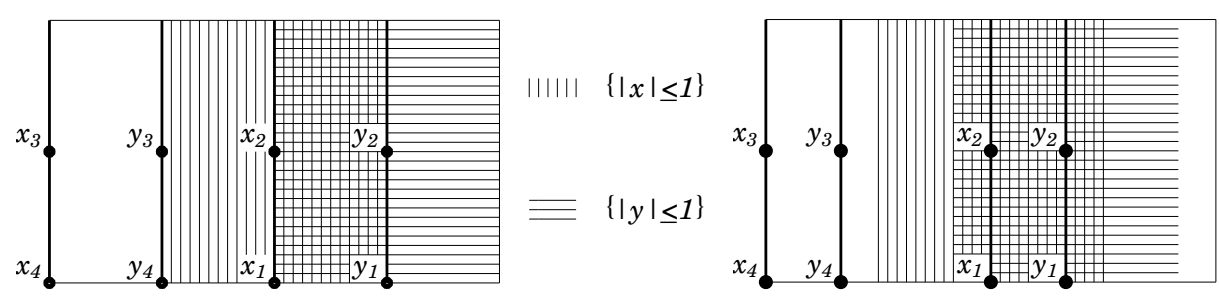

Fig. 4. Examples of the parallelogram $\left[0, \omega_{2}\left[\times\left[0, \omega_{1} / \imath[\right.\right.\right.$ with its important cycles, on the left for $\{N, E, S, W\}$ and on the right for $\{N, E, S E, S, W, N W\}$.

For the walk $\{N, E, S, W\}$, the group has indeed order 4, and Proposition 5 entails $\omega_{3}=\omega_{2} / 2$. Moreover, it is easy to show that in this case $X\left(\left[y_{1}, y_{2}\right]\right)=X\left(\left[y_{3}, y_{4}\right]\right)=$ $Y\left(\left[x_{1}, x_{2}\right]\right)=Y\left(\left[x_{3}, x_{4}\right]\right)$ coincides with the unit circle. In particular, $x^{-1}(\{|x|=1\})=$ $\left(\left[0, \omega_{1}\left[+\omega_{2} / 4\right) \cup\left(\left[0, \omega_{1}\left[+3 \omega_{2} / 4\right)\right.\right.\right.\right.$ and $y^{-1}(\{|y|=1\})=\left(\left[0, \omega_{1}[) \cup\left(\left[0, \omega_{1}\left[+\omega_{2} / 2\right)\right.\right.\right.\right.$. See Figure 4 (left).

Further, for the walk $\{N, E, S E, S, W, N W\}$, the group has order 6 and the covariance is negative, hence by Proposition 5 we obtain $\omega_{3}=\omega_{2} / 3$. Then by the same arguments as in the proof of [17, Proposition 26], we obtain the location of the cycles $x^{-1}(\{|x|=1\})$ and $y^{-1}\left(\{|y|=1\}\right.$ for this walk, namely, $x^{-1}(\{|x|=1\})=\left(\left[0, \omega_{1}\left[+\omega_{2} / 4\right) \cup\left(\left[0, \omega_{1}[+\right.\right.\right.\right.$ $\left.3 \omega_{2} / 4\right)$ and $y^{-1}\left(\{|y|=1\}=\left(\left[0, \omega_{1}\left[+5 \omega_{2} / 12\right) \cup\left(\left[0, \omega_{1}\left[+11 \omega_{2} / 12\right)\right.\right.\right.\right.\right.$. See Figure 4 (right). 


\section{Conformal gluing functions}

The main subject of this section is to introduce and to study suitable CGFs (see Definition 1) $w$ and $\widetilde{w}$ for the sets $\mathscr{G} X\left(\left[y_{1}, y_{2}\right]\right)$ and $\mathscr{G} Y\left(\left[x_{1}, x_{2}\right]\right)$ in all the 74 non-degenerate cases. For this purpose, we first find explicitly, in Subsection 6.1, all appropriate CGFs for both sets above (essentially thanks to the uniformization studied in Section 5), and in particular we prove Theorem 6 . Then we observe that the behavior of these CGFs is strongly influenced by the finiteness of the group $W$ defined in (5). Accordingly, we study separately, in Subsections 6.2 and 6.3, the walks having an infinite and then a finite group, and we show Theorems 2 and 3.

\subsection{Finding all suitable conformal gluing functions}

The book [9] provides explicitly one CGF, and it does so only for $z=1 / k$. We begin here by generalizing this result and by finding the expressions of all possible CGFs for the sets $\mathscr{G} X\left(\left[y_{1}, y_{2}\right]\right)$ and $\mathscr{G} Y\left(\left[x_{1}, x_{2}\right]\right)$ for any value of $z$. We start by quoting [9, p. 126]: if we denote $\widehat{w}=w \circ x$ or $\widehat{w}=w \circ y$ with $x, y$ defined in (16), then the problem of finding a CGF $w$ is equivalent to finding a function $\widehat{w}$ meromorphic in $\left[\omega_{2} / 2,\left(\omega_{2}+\omega_{3}\right) / 2\right] \times \mathbb{R}$, $\omega_{1}$-periodic, with only one simple pole in $\left[\omega_{2} / 2,\left(\omega_{2}+\omega_{3}\right) / 2\right] \times\left[0, \omega_{1} / l\right]$ (hatched in Figure 5, left), and satisfying the following two conditions:

(i) for all $\omega \in\left[-\omega_{1} / 2, \omega_{1} / 2\right], \widehat{w}\left(\left[\omega_{1}+\omega_{2}\right] / 2+\omega\right)=\widehat{w}\left(\left[\omega_{1}+\omega_{2}\right] / 2-\omega\right)$;

(ii) for all $\omega \in\left[-\omega_{1} / 2, \omega_{1} / 2\right], \widehat{w}\left(\left[\omega_{1}+\omega_{2}+\omega_{3}\right] / 2+\omega\right)=\widehat{w}\left(\left[\omega_{1}+\omega_{2}+\omega_{3}\right] / 2-\omega\right)$.

Setting $\bar{w}(\omega)=\widehat{w}\left(\left[\omega_{1}+\omega_{2}\right] / 2+\omega\right)$, the above problem becomes that of finding a function $\bar{w}$ meromorphic in $\left[0, \omega_{3} / 2\right] \times \mathbb{R}, \omega_{1}$-periodic, with only one simple pole in $\left[0, \omega_{3} / 2\right] \times\left[-\omega_{1} /(2 \imath), \omega_{1} /(2 \imath)\right]$, and such that:

(i') for all $\omega \in\left[-\omega_{1} / 2, \omega_{1} / 2\right], \bar{w}(\omega)=\bar{w}(-\omega)$;

(ii') for all $\omega \in\left[-\omega_{1} / 2, \omega_{1} / 2\right], \bar{w}\left(\omega_{3} / 2+\omega\right)=\bar{w}\left(\omega_{3} / 2-\omega\right)$.

Now we notice that by analytic continuation, $\left(i^{\prime}\right)$ allows us to continue the function $\bar{w}$ from $\left[0, \omega_{3} / 2\right] \times \mathbb{R}$ to $\left[-\omega_{3} / 2, \omega_{3} / 2\right] \times \mathbb{R}$, and next, also by analytic continuation, (ii') enables us to continue $\bar{w}$ as a $\omega_{3}$-periodic function-since evaluating (ii') at $\omega_{3} / 2+\omega$ and using (i') lead to $\bar{w}\left(\omega_{3}+\omega\right)=\bar{w}(\omega)$. In particular, the problem of finding the CGF finally becomes the following: find an even elliptic function $\bar{w}$ with periods $\omega_{1}, \omega_{3}$ with only two simple poles at $\pm p$ (or one double pole at $p$ if $p$ and $-p$ are congruent modulo the lattice) in the parallelogram $\left[-\omega_{3} / 2, \omega_{3} / 2\right] \times\left[-\omega_{1} /(2 \imath), \omega_{1} /(2 \imath)\right]$ (see Figure 5, right).

Let $\wp_{1,3}$ denote the Weierstrass elliptic function with periods $\omega_{1}, \omega_{3}$. A crucial fact for us is the following.

Lemma 6. Let $p \in\left[-\omega_{3} / 2, \omega_{3} / 2\right] \times\left[-\omega_{1} /(2 \imath), \omega_{1} /(2 \imath)\right]$.

- If $p=0$, the solutions of the problem above are $\left\{\alpha+\beta \wp_{1,3}(\omega): \alpha, \beta \in \mathbb{C}\right\}$.

- If $p \neq 0$, the solutions are $\left\{\alpha+\beta /\left[\wp_{1,3}(\omega)-\wp_{1,3}(p)\right]: \alpha, \beta \in \mathbb{C}\right\}$. 

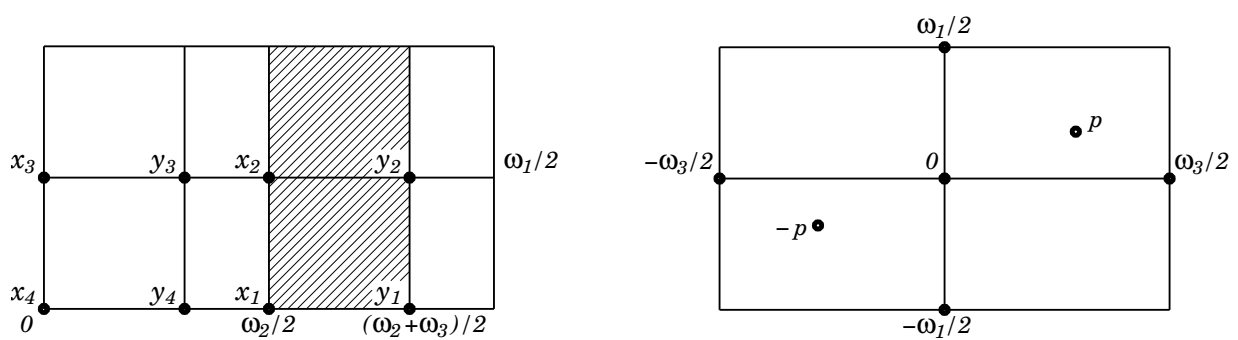

Fig. 5. Domains of definition of $\widehat{w}$ (left) and $\bar{w}$ (right).

Proof. It is well-known (see, e.g., [15, Theorems 3.11.1 and 3.13.1]) that an even elliptic function with periods $\omega_{1}, \omega_{3}$ having $2 q$ poles in a period parallelogram is necessarily a rational function of order $q$ of $\wp_{1,3}$. In our case, $\bar{w}$, which has exactly two poles of order one or one pole of order two in $\left[-\omega_{3} / 2, \omega_{3} / 2\right] \times\left[-\omega_{1} /(2 \imath), \omega_{1} /(2 \imath)\right]$, is thus a fractional linear transformation of $\wp 1,3$.

- In particular, it is immediate that $p=0$ yields $\bar{w}(\omega)=\alpha+\beta \wp_{1,3}(\omega)$.

- If $p \neq 0$ then $\wp_{1,3}(p) \neq \infty$, and we get

$$
\bar{w}(\omega)=\left[\alpha \wp_{1,3}(\omega)+\gamma\right] /\left[\wp_{1,3}(\omega)-\wp_{1,3}(p)\right] .
$$

Since by (16) we have $x(\omega)=f^{-1}(\wp(\omega))$ with $f$ defined in (18), applying Lemma 6 for $p=0$ yields the following theorem.

Theorem 6. The functions

$$
w(t)=\wp_{1,3}\left(\wp^{-1}(f(t))-\left[\omega_{1}+\omega_{2}\right] / 2\right), \quad \widetilde{w}(t)=w\left(X_{0}(t)\right)
$$

are suitable CGFs for the sets $\mathscr{G} X\left(\left[y_{1}, y_{2}\right]\right)$ and $\mathscr{G} Y\left(\left[x_{1}, x_{2}\right]\right)$, respectively.

Remark 5. By (16) we have $x(\omega)=X_{0}(y(\omega))$ so that, of course,

$$
\widetilde{w}(t)=\wp_{1,3}\left(y^{-1}(t)-\left[\omega_{1}+\omega_{2}\right] / 2\right) .
$$

The CGF $w$ is therefore equal to the Weierstrass elliptic function with periods $\omega_{1}, \omega_{3}$ evaluated at a translation of the inverse of the Weierstrass elliptic function with periods $\omega_{1}, \omega_{2}$. It turns out that the theory of transformation of elliptic functions-the basic result of which is here recalled in (22) below-entails that this expression admits a wonderful simplification if $\omega_{3} / \omega_{2}$ is rational.

But Proposition 4 shows that the latter condition is related to the group $W=\langle\Psi, \Phi\rangle$ defined in (5), since it states that $\omega_{3} / \omega_{2}$ is rational for all $\left.z \in\right] 0,1 / k$ [ if and only if the group $W$ is finite. Consequently, we study $w$ separately, in Subsections 6.2 and 6.3 below, according to the finiteness of this group. It is worth noting that, to the best of our knowledge, these results on the CGFs are new, even for $z=1 / k$. 
Remark 6. Given a CGF $u$ for a generic set $\mathscr{C}$, any of its fractional linear transformations $[\alpha u+\beta] /[\gamma u+\delta]$ with $\alpha, \beta, \gamma, \delta \in \mathbb{C}$ such that $\alpha, \gamma \neq 0$ is also a CGF for $\mathscr{C}$ (see Definition 1). ${ }^{6}$ They are actually the only ones (see [19, Chapter 2] for a proof using Fredholm operators). Applying Lemma 6 to any value of $p$ enables us to recover this in an elementary way. This also has two interesting consequences. First, it means that Theorem 6 gives an explicit expression for all possible CGFs. Also, it implies that Theorem 2 can be extended to any CGFs; in other words, the nature of the CGFs is intrinsic, in the sense that it only depends on the kernel.

Remark 7. We recall from [18, Section 4] the following global properties of the CGFs. The function $w$ (resp. $\widetilde{w}$ ) defined by (21) is meromorphic on $\mathbb{C} \backslash\left[x_{3}, x_{4}\right]$ (resp. $\left.\mathbb{C} \backslash\left[y_{3}, y_{4}\right]\right)$. It has there one simple pole, at $x_{2}$ (resp. $\left.Y\left(x_{2}\right)\right)$, and $\left\lfloor\omega_{2} /\left(2 \omega_{3}\right)\right\rfloor^{7}$ double poles at some points lying on the segment $] x_{2}, x_{3}\left[\cap\left(\mathbb{C} \backslash \mathscr{G} X\left(\left[y_{1}, y_{2}\right]\right)\right)\right.$ (resp. $] y_{2}, y_{3}[$ $\left.\cap\left(\mathbb{C} \backslash \mathscr{G} Y\left(\left[x_{1}, x_{2}\right]\right)\right)\right)$.

Before concluding this part, we state the following properties of the $\wp$-Weierstrass function, which will be of the highest significance for the proof of Theorem 3 .

Lemma 7. The following results on the $\wp$-Weierstrass elliptic function hold:

- Let $\wp$ be a Weierstrass elliptic function with periods $\bar{\omega} \widehat{\omega}$, and let $p$ be a positive integer. The Weierstrass elliptic function with periods $\bar{\omega}, \widehat{\omega} / p$ can be written in terms of $\wp$ as

$$
\wp(\omega)+\sum_{k=1}^{p-1}[\wp(\omega+k \widehat{\omega} / p)-\wp(k \widehat{\omega} / p)] .
$$

- Let $\wp$ be a Weierstrass elliptic function. We have the addition theorem:

$$
\forall \omega, \widetilde{\omega}, \quad \wp(\omega+\widetilde{\omega})=-\wp(\omega)-\wp(\widetilde{\omega})+\frac{1}{4}\left[\frac{\wp^{\prime}(\omega)-\wp^{\prime}(\widetilde{\omega})}{\wp(\omega)-\wp(\widetilde{\omega})}\right]^{2} .
$$

- For any integer $r \geq 0$, there is a polynomial $p_{r}(x)$, with main term $(2 r+1) ! x^{r+1}$, such that

$$
\wp^{(2 r)}=p_{r}(\wp)
$$

Proof. Equality (22) is shown, e.g., in [24, p. 456]. Identity (23) is classical, and can be found in $[15,24]$. As for (24), differentiating (17) gives $\wp^{\prime \prime}=6 \wp^{2}-g_{2} / 2$ (with obvious notations), and higher derivatives of even order are clearly expressible as in (24).

\footnotetext{
6 Incidentally, we note that the quantity $\partial_{t} u(t) /[u(t)-u(x)]$ appearing in Theorem 1 is invariant under the transformation $u \mapsto[\alpha u+\beta] /[\gamma u+\delta]$, as soon as $\alpha, \gamma \neq 0$.

${ }^{7}$ For $r \in \mathbb{R},\lfloor r\rfloor$ denotes the lower integer part of $r$, i.e., the unique $p \in \mathbb{Z}$ such that $p \leq r<$ $p+1$.
} 


\subsection{Case of an infinite group}

In this subsection we concentrate on the case of an infinite group of the walk, and we prove the part of Theorem 2 concerning the infinite group case. Precisely, we show that for any $z \in] 0,1 / k$ [ such that $\omega_{3} / \omega_{2}$ is irrational, the CGFs $w$ and $\widetilde{w}$ defined in (21) are non-holonomic. Before starting the proof, we notice that for a walk admitting an infinite group, it may happen that $\omega_{3} / \omega_{2}$ is rational for certain values of $z$, but $\omega_{3} / \omega_{2}$ also has to take irrational values. Indeed, if $\omega_{3} / \omega_{2}$ were rational for all $\left.z \in\right] 0,1 / k\left[\right.$, then $\omega_{3} / \omega_{2}$ would be a rational constant, since by (15) and (19), the quantity $\omega_{3} / \omega_{2}$ is holomorphic in $z$, and Proposition 4 would then entail that the group is finite.

Proof of Theorem 2 (infinite group case). Let

$$
v(t)=w\left(f^{-1}(t)\right)=\wp_{1,3}\left(\wp^{-1}(t)-\left[\omega_{1}+\omega_{2}\right] / 2\right) .
$$

The class of holonomic functions being closed under algebraic substitutions (see, e.g., [11]), it is enough to prove that $v$ is non-holonomic. Indeed, on the one hand $f$ is rational, and on the other hand $\widetilde{w}=w\left(X_{0}\right)$, where $X_{0}$ is algebraic. We are going to show first that $v$ is non-algebraic, and then we will prove that if $v$ is holonomic then it has to be algebraic, so that $v$ will be non-holonomic.

Suppose thus that $v$ is algebraic. In other words, we assume that there exist polynomials $a_{0}, \ldots, a_{q}$ with $a_{q} \neq 0$ such that $\sum_{k=0}^{q} a_{k}(t) v(t)^{k}=0$. By the definition of $v$ and since the Weierstrass elliptic function is non-algebraic, at least one of $a_{0}, \ldots, a_{q}$ is nonconstant. Evaluating the last equality at $t=\wp\left(\omega+\left[\omega_{1}+\omega_{2}\right] / 2\right)$ and using the definition of $v$, we get $\sum_{k=0}^{q} a_{k}\left(\wp\left(\omega+\left[\omega_{1}+\omega_{2}\right] / 2\right)\right) \wp_{1,3}(\omega)^{k}=0$. Since $\wp\left(\omega+\left[\omega_{1}+\omega_{2}\right] / 2\right)$ is a rational transformation of $\wp(\omega)$ (see the addition theorem (23)), the last equality yields the identity $P\left(\wp(\omega), \wp_{1,3}(\omega)\right)=0$, where $P$ is a certain polynomial, which is non-constant with respect to the two variables.

Now we recall $[15,24]$ that $\wp$ (resp. $\left.\wp_{1,3}\right)$ has poles at every point of the lattice $\omega_{1} \mathbb{Z}+\omega_{2} \mathbb{Z}$ (resp. $\omega_{1} \mathbb{Z}+\omega_{3} \mathbb{Z}$ ). Moreover, it is well known that $\omega_{2} \mathbb{Z}+\omega_{3} \mathbb{Z}$ is dense in $\mathbb{R}$ if $\omega_{3} / \omega_{2}$ is irrational. In particular, if $\omega_{3} / \omega_{2}$ is irrational then the poles of $P\left(\wp(\omega), \wp_{1,3}(\omega)\right)$ are not isolated, which contradicts the principle of analytic continuation.

Suppose now that $v$ is holonomic, i.e., there exist polynomials $a_{0}, \ldots, a_{q}$ with $a_{q} \neq 0$ such that $\sum_{k=0}^{q} a_{k}(t) v^{(k)}(t)=0$. We show that, in this case,

$$
\sum_{k=0}^{q} a_{k}(t) v^{(k)}(t)=U(t, v(t))+V(t, v(t)) v^{\prime}(t),
$$

where the dependence of $U, V$ on the first (resp. second) variable is rational (resp. polynomial), and where at least one of $U, V$ is non-zero. These facts will entail the algebraicity of $v$. Indeed:

- If $V$ is identically zero, then $U$ has to be non-zero. Moreover, since $U$ is rational with respect to the first variable and since by $(25)$ we have $U(t, v(t))=0, U$ has to be non-constant with respect to the second variable, and (25) immediately implies that $v$ is then algebraic. 
- Suppose now that $V$ is not identically zero. From (25) and the supposed holonomicity of $v$ it follows that $U(t, v(t))^{2}-V(t, v(t))^{2} v^{\prime}(t)^{2}=0$. Also, denoting by $g$ the derivative of $\wp^{-1}$ (if $g_{2}$ and $g_{3}$ are the invariants ${ }^{8}$ of $\wp$, then we have $g(t)=$ $\left.1 /\left[4 t^{3}-g_{2} t-g_{3}\right]^{1 / 2}\right)$, we obtain $v^{\prime}(t)^{2}=g(t)^{2} \wp_{1,3}^{\prime}\left(\wp^{-1}(t)-\left[\omega_{1}+\omega_{2}\right] / 2\right)^{2}$. But $\wp_{1,3}^{\prime 2}=4 \wp_{1,3}^{3}-g_{2,1,3} \wp_{1,3}-g_{3,1,3}$, where $g_{2,1,3}$ and $g_{3,1,3}$ are the invariants of $\wp_{1,3}$. Finally, we have $U(t, v(t))^{2}-V(t, v(t))^{2} g(t)^{2}\left[4 v(t)^{3}-g_{2,1,3} v(t)-g_{3,1,3}\right]=0$. This last quantity is a rational function of the first variable, since $g(t)^{2}$ is rational, and an odd-degree polynomial in the second one, so that $v$ is algebraic.

So, it is definitely enough to prove (25). For this we are going to show that for any $k \geq 0$,

$$
v^{(k)}(t)=U_{k}(t, v(t))+V_{k}(t, v(t)) v^{\prime}(t),
$$

where $U_{k}$ (resp. $V_{k}$ ) is rational with respect to the first variable and a polynomial of degree exactly $\lfloor k / 2+1\rfloor$ (resp. $\lfloor(k-1) / 2\rfloor)$ with respect to the second one. Equality (25) will then be an immediate consequence of (26). Indeed, if $q$ is even then by using (26) in (25) we find that the degree of $U$ in $v$ is exactly $\lfloor q / 2+1\rfloor$ and $U$ is thus obviously non-zero. Likewise, if $q$ is odd we infer that the degree of $V$ is exactly $\lfloor(q-1) / 2\rfloor$ and is thus clearly non-zero if $q \geq 3$. If $q=1$ then we easily specify $V_{0}(=0)$ and $V_{1}$, and we immediately deduce that $V$ is also non-zero.

We now show (26). For $k=0$, this is obvious. For $k \geq 1$, a straightforward calculation leads to

$$
v^{(k)}(t)=\sum_{p=1}^{k} b_{p}(t) \wp_{1,3}^{(p)}\left(\wp^{-1}(t)-\left[\omega_{1}+\omega_{2}\right] / 2\right)
$$

with $b_{k}=g(t)^{k}, b_{k-1}(t)=[k(k-1) / 2] g^{\prime}(t) g(t)^{k-2}$, and for $p \in\{2, \ldots, k-1\}, b_{k-p}$ is a polynomial in the variables $g(t), g^{\prime}(t), \ldots, g^{(p)}(t)$. Moreover, if $p$ is even then $b_{p}$ is clearly rational, whereas if $p$ is odd then $b_{p} / g$ is rational. Next, by repeated application of $(24)$ and by using that $\wp_{1,3}^{\prime}\left(\wp^{-1}(t)-\left[\omega_{1}+\omega_{2}\right] / 2\right)=v^{\prime}(t) / g(t)$, we obtain

$$
v^{(k)}(t)=\sum_{\substack{p=1 \\ p \text { even }}}^{k} b_{p}(t) p_{p / 2}(v(t))+v^{\prime}(t) \sum_{\substack{p=1 \\ p \text { odd }}}^{k}\left[b_{p}(t) / g(t)\right] p_{(p-1) / 2}^{\prime}(v(t)) .
$$

With the values of $b_{k}$ and $b_{k-1}$ given above, this immediately yields (26), and therefore also (25), and finally the fact that $v, w$ and $\widetilde{w}$ are non-holonomic.

\subsection{Case of a finite group}

In this subsection, we consider the case of a finite group. We show that $w$ and $\widetilde{w}$ are then algebraic, and we complete the proof of Theorem 2 . Moreover, we considerably simplify the explicit expressions of $w$ and $\widetilde{w}$ given in (21) for 22 of the 23 walks having a finite

\footnotetext{
8 They are defined by $g_{2}=-4\left[\wp\left(\omega_{1} / 2\right) \wp\left(\left[\omega_{1}+\omega_{2}\right] / 2\right)+\wp\left(\omega_{1} / 2\right) \wp\left(\omega_{2} / 2\right)+\wp\left(\left[\omega_{1}+\right.\right.\right.$ $\left.\left.\left.\omega_{2}\right] / 2\right) \wp\left(\omega_{2} / 2\right)\right]$ and $g_{3}=4 \wp\left(\omega_{1} / 2\right) \wp\left(\left[\omega_{1}+\omega_{2}\right] / 2\right) \wp\left(\omega_{2} / 2\right)$.
} 
group: ${ }^{9}$ see Theorem 3(i) (resp. (ii) and (iii), (iv)) for the walks admitting a group of order 4 (resp. 6, 8). Thanks to Proposition 4 , we conclude that for the 23 walks having a finite group, the quantity $\omega_{2} / \omega_{3}$ is rational. Moreover, by [6] and Proposition 5, it is sufficient to consider the cases where $\omega_{2} / \omega_{3}$ is equal to $2,3,3 / 2,4,4 / 3$. According to the classification of [6] - recalled here in Figure 6-there are 16, 2, 3, 1, 1 such walks, respectively.

Finite group of the walk and negative or zero covariance. We first consider the case of covariance equal to zero-or equivalently the case $\omega_{2} / \omega_{3}=2$.

Proof of Theorem 3(i). Actually, it suffices to show the following identity (specific to the case $\left.\omega_{2} / \omega_{3}=2\right)$ :

$\wp_{1,3}\left(\omega-\left[\omega_{1}+\omega_{2}\right] / 2\right)=-2 f\left(x_{1}\right)+\left[f\left(x_{2}\right)-f\left(x_{3}\right)\right]^{2} \frac{\wp(\omega)-f\left(x_{1}\right)}{\left[\wp(\omega)-f\left(x_{2}\right)\right]\left[\wp(\omega)-f\left(x_{3}\right)\right]}$.

Indeed, if (27) holds, let us evaluate it at $\omega=\wp^{-1}(f(t))$ : by (21) there exist constants $K_{1}$ and $K_{2}$ such that $w(t)=K_{1}+K_{2}\left[f(t)-f\left(x_{1}\right)\right] /\left[\left(f(t)-f\left(x_{2}\right)\right)\left(f(t)-f\left(x_{3}\right)\right)\right]$. But by using the explicit expression (18) of $f$, it is immediate that if $x_{4} \neq \infty$ then $w(t)=K_{1}+K_{3}\left[\left(t-x_{1}\right)\left(t-x_{4}\right)\right] /\left[\left(t-x_{2}\right)\left(t-x_{3}\right)\right]$, and if $x_{4}=\infty$ then $w(t)=$ $K_{1}+K_{4}\left[t-x_{1}\right] /\left[\left(t-x_{2}\right)\left(t-x_{3}\right)\right], K_{3}$ and $K_{4}$ being some non-zero constants.

To prove (27), we start by applying (22) for $p=2$ : we obtain

$$
\wp 1,3\left(\omega-\left[\omega_{1}+\omega_{2}\right] / 2\right)=\wp\left(\omega-\left[\omega_{1}+\omega_{2}\right] / 2\right)+\wp\left(\omega-\omega_{1} / 2\right)-\wp\left(\omega_{2} / 2\right) .
$$

Now, taking the usual notations $e_{1}=\wp\left(\omega_{1} / 2\right), e_{1+2}=\wp\left(\left[\omega_{1}+\omega_{2}\right] / 2\right)$ and $e_{2}=$ $\wp\left(\omega_{2} / 2\right)$, we can state the following two particular cases of the addition formula (23):

$$
\begin{aligned}
\wp\left(\omega-\left[\omega_{1}+\omega_{2}\right] / 2\right) & =e_{1+2}+\left[\left(e_{1+2}-e_{1}\right)\left(e_{1+2}-e_{2}\right)\right] /\left[\wp(\omega)-e_{1+2}\right], \\
\wp\left(\omega-\omega_{1} / 2\right) & =e_{1}+\left[\left(e_{1}-e_{2}\right)\left(e_{1}-e_{1+2}\right)\right] /\left[\wp(\omega)-e_{1}\right] .
\end{aligned}
$$

In this way and after simplification we obtain

$$
\begin{aligned}
& \wp_{1,3}\left(\omega-\left[\omega_{1}+\omega_{2}\right] / 2\right) \\
& \quad=e_{1}-e_{2}+e_{1+2}+\left(e_{1}-e_{1+2}\right)^{2}\left[\wp(\omega)-e_{2}\right] /\left[\left(\wp(\omega)-e_{1}\right)\left(\wp(\omega)-e_{1+2}\right)\right] .
\end{aligned}
$$

Finally, by using the equalities $e_{1}=f\left(x_{3}\right), e_{1+2}=f\left(x_{2}\right)$ and $e_{2}=f\left(x_{1}\right)$ (see below (18)), as well as $e_{1}+e_{1+2}+e_{2}=0$, we immediately obtain (27).

Let us now consider the case of negative covariance-or equivalently $\omega_{2} / \omega_{3}$ equal to 3 or 4 . We start with the situation where $\omega_{2} / \omega_{3}=3$.

Proof of Theorem 3(ii). First of all, let us give a sketch of the proof. We shall first show the existence and find expressions for two third-degree polynomials $A$ and $B$ such that $\wp_{1,3}\left(\omega-\left[\omega_{1}+\omega_{2}\right] / 2\right)=A(\wp(\omega)) / B(\wp(\omega))$. In particular, by $(21)$, we shall then infer that $w(t)=A(f(t)) / B(f(t))$. Next we shall consider the two walks $\{\mathrm{N}, \mathrm{SE}, \mathrm{W}\}$ and $\{\mathrm{N}, \mathrm{E}, \mathrm{SE}, \mathrm{S}, \mathrm{W}, \mathrm{NW}\}$ separately.

\footnotetext{
9 The 23rd model—namely, Gessel's walk—has already been treated in [17].
} 
- For the walk $\{\mathrm{N}, \mathrm{SE}, \mathrm{W}\}$, we have $x_{4}=\infty$ and so $A(f(t))$ and $B(f(t))$ are also third-degree polynomials (see the expression (18) of $f$ ). We will show that $B(f(t))=$ $\left(t-x_{2}\right)\left(t-1 / x_{2}^{1 / 2}\right)^{2}$. In addition, if $r(t)$ denotes the remainder of euclidean division of $A(f(t))$ by $B(f(t))$, we will prove that $r(t)=\left(r^{\prime \prime}(0) / 2\right) t^{2}$ with $r^{\prime \prime}(0) \neq 0$, so that

$$
w(t)=B(f(\infty)) / A(f(\infty))+\left(r^{\prime \prime}(0) / 2\right) t^{2} /\left[\left(t-x_{2}\right)\left(t-1 / x_{2}^{1 / 2}\right)^{2}\right] .
$$

- For the walk $\{\mathrm{N}, \mathrm{E}, \mathrm{SE}, \mathrm{S}, \mathrm{W}, \mathrm{NW}\}$, we have $x_{4} \neq \infty$ and so $\left(t-x_{4}\right)^{3} A(f(t))$ and $\left(t-x_{4}\right)^{3} B(f(t))$ are third-degree polynomials. We will show that $\left(t-x_{4}\right)^{3} B(f(t))=$ $\left(t-x_{2}\right)\left(t-1 / x_{2}{ }^{1 / 2}\right)^{2}$. If we denote by $r(t)$ the remainder of euclidean division of $\left(t-x_{4}\right)^{3} A(f(t))$ by $\left(t-x_{4}\right)^{3} B(f(t))$, then we will prove that $r(t)=\left(r^{\prime \prime}(0) / 2\right) t(t+1)$ with $r^{\prime \prime}(0) \neq 0$ so that

$$
w(t)=B(f(\infty)) / A(f(\infty))+\left(r^{\prime \prime}(0) / 2\right) t(t+1) /\left[\left(t-x_{2}\right)\left(t-1 / x_{2}^{1 / 2}\right)^{2}\right] .
$$

Finally, with the explicit formula for $w$ and the fact that $\widetilde{w}=w\left(X_{0}\right)$, an elementary calculation will lead, for each walk, to the expression of $\widetilde{w}$ stated in Theorem 3(ii); this will conclude the proof.

So we begin by finding explicitly, for both $\{N, S E, W\}$ and $\{N, E, S E, S, W, N W\}$, two polynomials $A$ and $B$ of degree three such that $\wp_{1,3}\left(\omega-\left[\omega_{1}+\omega_{2}\right] / 2\right)=$ $A(\wp(\omega)) / B(\wp(\omega))$.

Applying (22) with $p=3$, we get

$$
\begin{aligned}
\wp 1,3\left(\omega-\left[\omega_{1}+\omega_{2}\right] / 2\right)= & \wp\left(\omega-\left[\omega_{1}+\omega_{2}\right] / 2\right)+\wp\left(\omega-\omega_{1} / 2-\omega_{2} / 6\right)-\wp\left(\omega_{2} / 3\right) \\
& +\wp\left(\omega-\omega_{1} / 2+\omega_{2} / 6\right)-\wp\left(2 \omega_{2} / 3\right) .
\end{aligned}
$$

Then, using the addition formula (23) for $\wp$, setting $K=e_{1+2}-2 \wp\left(\omega_{2} / 3\right)-$ $2 \wp\left(\omega_{1} / 2+\omega_{2} / 6\right)$, using that $\wp\left(\omega_{1} / 2+\omega_{2} / 6\right)=\wp\left(\omega_{1} / 2-\omega_{2} / 6\right)$ and $\wp^{\prime}\left(\omega_{1} / 2+\omega_{2} / 6\right)=$ $-\wp^{\prime}\left(\omega_{1} / 2-\omega_{2} / 6\right)$ (following from the fact that $\wp\left(\omega_{1} / 2+\omega\right)$ is even and $\wp^{\prime}\left(\omega_{1} / 2+\omega\right)$ is odd $)$ we find that $\wp_{1,3}\left(\omega-\left[\omega_{1}+\omega_{2}\right] / 2\right)$ equals

$$
\frac{\left(e_{1+2}-e_{1}\right)\left(e_{1+2}-e_{2}\right)}{\wp(\omega)-e_{1+2}}-2 \wp(\omega)+\frac{1}{2} \frac{\wp^{\prime}(\omega)^{2}+\wp^{\prime}\left(\omega_{1} / 2+\omega_{2} / 6\right)^{2}}{\left[\wp(\omega)-\wp\left(\omega_{1} / 2+\omega_{2} / 6\right)\right]^{2}}+K .
$$

From (17) and (28), it is now clear that $\wp_{1,3}\left(\omega-\left[\omega_{1}+\omega_{2}\right] / 2\right)$ can be written as $A(\wp(\omega)) / B(\wp(\omega))$; moreover we can take $B(\wp(\omega))=\left(\wp(\omega)-e_{1+2}\right)(\wp(\omega)-$ $\left.\wp\left(\omega_{1} / 2+\omega_{2} / 6\right)\right)^{2}$.

Now we show that $\wp\left(\omega_{1} / 2+\omega_{2} / 6\right)=f\left(1 / x_{2}^{1 / 2}\right)$. For this we will express $\wp\left(\omega_{1} / 2+\right.$ $\left.\omega_{2} / 6\right)$ in terms of $z$. Since $\wp\left(\omega_{1} / 2+\omega_{2} / 6\right)=e_{1}+\left[\left(e_{1}-e_{2}\right)\left(e_{1}-e_{1+2}\right)\right] /\left[\wp\left(\omega_{2} / 6\right)-e_{1}\right]$ it is enough to express $\wp\left(\omega_{2} / 6\right)$ in terms of $z$. For this we will first find $\wp\left(\omega_{2} / 3\right)$ explicitly and then use, for $\omega=\omega_{2} / 3$, the fact that $\wp(\omega / 2)$ is equal to

$$
\begin{aligned}
\wp(\omega)+[ & \left.\left(\wp(\omega)-e_{1}\right)\left(\wp(\omega)-e_{2}\right)\right]^{1 / 2} \\
+ & {\left[\left(\wp(\omega)-e_{1}\right)\left(\wp(\omega)-e_{1+2}\right)\right]^{1 / 2}+\left[\left(\wp(\omega)-e_{2}\right)\left(\wp(\omega)-e_{1+2}\right)\right]^{1 / 2} }
\end{aligned}
$$


(see $[15,24])$. In other words, for all coefficients in (28) to be explicit with respect to $z$, it is enough to find only $\wp\left(\omega_{2} / 3\right)$ in terms of $z$.

And now we show that for both $\{N, S E, W\}$ and $\{N, E, S E, S, W, N W\}, \wp\left(\omega_{2} / 3\right)=$ $1 / 3$. For this we use the following fact, already recalled in [17]: the quantity $x=\wp\left(\omega_{2} / 3\right)$ is the only positive root of

$$
x^{4}-g_{2} x^{2} / 2-g_{3} x-g_{2}^{2} / 48,
$$

with $g_{2}=-4\left[e_{1} e_{2}+e_{1} e_{1+2}+e_{2} e_{1+2}\right]$ and $g_{3}=4 e_{1} e_{2} e_{1+2}$ being the invariants of $\wp$. By using the explicit expressions of $e_{1}, e_{2}$ and $e_{1+2}$-see the proof of Theorem 3(i)we easily show that $1 / 3$ is a root of the polynomial above; $1 / 3$ being positive, we get $\wp\left(\omega_{2} / 3\right)=1 / 3$.

Then an elementary calculation leads to $\wp\left(\omega_{1} / 2+\omega_{2} / 6\right)=f\left(1 / x_{2}^{1 / 2}\right)$. Next from (17) we also obtain $\wp^{\prime}\left(\omega_{1} / 2+\omega_{2} / 6\right)$ and thus all coefficients in (28) are known in terms of $z$. In particular, this is also the case for the polynomials $A$ and $B$. After a lengthy but easy calculation we obtain the facts claimed in the items above, and thus Theorem 3(ii).

Proof of Theorem 3(iv). This case, concerning $\omega_{2} / \omega_{3}=4$, could be obtained by applying (22) for $p=4$; the details would be essentially the same as above, so we omit them.

Finite group of the walk and positive covariance. The only such possible walks are $\{N E, S, W\},\{N, E, S W\},\{N, N E, E, S, S W, W\}$, as well as Gessel's walk $\{E, S W, W, N E\}$. The latter has already been considered in [17]: there we have shown that the CGFs $w$ and $\widetilde{w}$ defined by (21) are algebraic (of degree three in $t$ ), and we have found their minimal polynomials.

By using the same key idea as in [17] —namely, a double application of (22) —we are now going to prove Theorem 3(iii), i.e., to show that for the three walks $\{N E, S, W\}$, $\{\mathrm{N}, \mathrm{E}, \mathrm{SW}\}$ and $\{\mathrm{N}, \mathrm{NE}, \mathrm{E}, \mathrm{S}, \mathrm{SW}, \mathrm{W}\}, w$ and $\widetilde{w}$ are algebraic (of degree two in $t$ ), and to find their minimal polynomials.

Proof of Theorem 3(iii). First recall that for the three walks considered here $\omega_{2} / \omega_{3}=$ $3 / 2$, and define the auxiliary period $\omega_{4}=\omega_{2} / 3$.

First, using $\omega_{4}=\omega_{2} / 3$ and (22), we shall be able to express $\wp_{1,4}$ as a rational function of $\wp$. Moreover, since $\omega_{4}=\omega_{3} / 2$ and once again applying (22), we shall write $\wp_{1,4}$ as a rational function of $\wp_{1,3}$. As an immediate consequence, $\wp_{1,3}$ will be an algebraic function of $\wp$. Then, applying (21) and the addition formula (23), we shall conclude that the CGF $w$ defines an algebraic function of $t$.

Rational expression of $\wp_{1,4}$ in terms of $\wp$. By using exactly the same arguments as in the proof of Theorem 3(ii), we obtain the following three facts: firstly, $\wp_{1,4}\left(\omega-\left[\omega_{1}+\omega_{2}\right] / 2\right)$ is equal to (28); secondly, $\wp\left(\omega_{2} / 3\right)=1 / 3$; and thirdly, the expressions of all coefficients in (28) with respect to $z$ are explicit. This way, we conclude that there exist $K_{1}$ and $K_{2}$ which depend only on $z$ - and could be made explicit—such that

$$
\wp_{1,4}\left(x^{-1}(t)-\left[\omega_{1}+\omega_{2}\right] / 2\right)=K_{1}+\frac{K_{2} u(t)}{\left[t-x_{2}\right]\left[t-1 / x_{2}{ }^{1 / 2}\right]^{2}},
$$

with $u(t)$ as described in the statement of Theorem 3(iii). 
Rational expression of $\wp_{1,4}$ in terms of $\wp_{1,3}$. Applying, as in the proof of Theorem 3(i), the identity (22) for $p=2$, we find that $\wp_{1,4}(\omega)=\wp_{1,3}(\omega)+\wp_{1,3}\left(\omega+\omega_{3} / 2\right)-\wp_{1,3}\left(\omega_{3} / 2\right)$. Setting then $e_{1,1,3}=\wp_{1,3}\left(\omega_{1} / 2\right), e_{1+3,1,3}=\wp_{1,3}\left(\left[\omega_{1}+\omega_{3}\right] / 2\right)$ and $e_{3,1,3}=\wp_{1,3}\left(\omega_{3} / 2\right)$ we have

$$
\wp_{1,3}\left(\omega+\omega_{3} / 2\right)=e_{3,1,3}+\left[\left(e_{3,1,3}-e_{1,1,3}\right)\left(e_{3,1,3}-e_{1+3,1,3}\right)\right] /\left[\wp_{1,3}(\omega)-e_{3,1,3}\right] .
$$

In particular, we immediately obtain

$$
\begin{aligned}
\wp_{1,3}(\omega)^{2}-\left[e_{3,1,3}+\right. & \left.\wp_{1,4}(\omega)\right] \wp_{1,3}(\omega) \\
& +\left[\left(e_{3,1,3}-e_{1,1,3}\right)\left(e_{3,1,3}-e_{1+3,1,3}\right)+e_{3,1,3} \wp_{1,4}(\omega)\right]=0 .
\end{aligned}
$$

Therefore, once the expressions of $e_{1,1,3}, e_{1+3,1,3}$ and $e_{3,1,3}$ are known explicitly, equations (21), (29) and (30) will immediately entail Theorem 3(iii).

It thus remains for us to find explicitly $e_{1,1,3}, e_{1+3,1,3}$ and $e_{3,1,3}$. This will be a consequence of the possibility of expanding $\wp_{1,4}$ in two different ways.

First, we saw above that

$$
\wp_{1,4}(\omega)=\wp_{1,3}(\omega)+\left[\left(e_{3,1,3}-e_{1,1,3}\right)\left(e_{3,1,3}-e_{1+3,1,3}\right)\right] /\left[\wp_{1,3}(\omega)-e_{3,1,3}\right],
$$

so that by using the expansion of $\wp_{1,3}$ at 0 , namely, $\wp_{1,3}(\omega)=1 / \omega^{2}+g_{2,1,3} \omega^{2} / 20+$ $g_{3,1,3} \omega^{4} / 28+O\left(\omega^{6}\right), g_{2,1,3}$ and $g_{3,1,3}$ being the invariants of $\wp_{1,3}$, as well as the straightforward equality $\left(e_{3,1,3}-e_{1,1,3}\right)\left(e_{3,1,3}-e_{1+3,1,3}\right)=3 e_{3,1,3}^{2}-g_{2,1,3} / 4$, we get

$$
\begin{aligned}
\wp_{1,4}(\omega)= & 1 / \omega^{2}+\left[3 e_{3,1,3}^{2}-g_{2,1,3} / 5\right] \omega^{2} \\
& +\left[g_{3,1,3}+3 e_{3,1,3}{ }^{3}-g_{2,1,3} e_{3,1,3} / 4\right] \omega^{4}+O\left(\omega^{6}\right) .
\end{aligned}
$$

Second, by applying the identity (22) for $p=3$ we obtain

$\wp_{1,4}(\omega)=-\wp(\omega)+\left[\wp^{\prime}(\omega)^{2}+\wp^{\prime}\left(\omega_{2} / 3\right)^{2}\right] /\left[2\left(\wp(\omega)-\wp\left(\omega_{2} / 3\right)\right)\right]-4 \wp\left(\omega_{2} / 3\right)$.

Using $\wp\left(\omega_{2} / 3\right)=1 / 3$ as well as (17) yields

$$
\wp_{1,4}(\omega)=1 / \omega^{2}+\left[2 / 3-9 g_{2} / 20\right] \omega^{2}+\left[10 / 27-g_{2} / 2-27 g_{3} / 28\right] \omega^{4}+O\left(\omega^{6}\right) .
$$

By identifying the expansions (31) and (32) we obtain expressions of $g_{2,1,3}$ and $g_{3,1,3}$ in terms of $g_{2}, g_{3}$ and $e_{3,1,3}$.

In addition, $e_{3,1,3}$ is obviously a solution of $4 e_{3,1,3}^{3}-g_{2,1,3} e_{3,1,3}-g_{3,1,3}=0$. If we use the expressions of $g_{2,1,3}$ and $g_{3,1,3}$ obtained just above, we conclude that

$$
e_{3,1,3}^{3}+\left[9 g_{2} / 16-5 / 6\right] e_{3,1,3}+\left[35 / 108-27 g_{3} / 32-7 g_{2} / 16\right]=0 \text {. }
$$

We can solve this equation (we recall that $g_{2}$ and $g_{3}$ are known explicitly with respect to $z$ ) and, this way, we get $e_{3,1,3}$. Next we obtain $g_{2,1,3}$ and $g_{3,1,3}$, or, equivalently, $e_{1,1,3}$ and $e_{1+3,1,3}$. In particular, the expansion (30) is now completely known and Theorem 3(iii) is proved.

\section{Conclusions and perspectives}

To conclude, we would like to mention some open problems and perspectives related to this paper. 
— In the finite group case, finding the way to generate a vanishing differential equation for the counting function starting from Theorem 1 is an open problem; on the other hand, it is certainly possible to verify a posteriori that the function satisfies a certain differential equation.

- In the infinite group case, it is an open problem to find the asymptotics, as $n \rightarrow \infty$, of $q_{i, j, n}$; see in particular the conjectures of Bostan and Kauers [3] on the total numbers of walks $\sum_{i, j \geq 0} q_{i, j, n}$. The singularity analysis of the expression of $Q(x, y ; z)$ obtained in Theorem 1—generically delicate, see [22, Chapter F]—should lead to these results.

- Last but not least, proving from Theorem 1 the conjecture of Bousquet-Mélou and Mishna—namely, that in the infinite groups case, $Q(x, y ; z)$ is non-holonomic-is also an open problem. However, everything suggests that this conjecture is truelike the fact that all known examples of infinite group [1,21] have non-holonomic counting functions, or else the link, suggested by Figure 6, between the nature of $Q(x, y ; z)$ and that of $w(x ; z)$ and $\widetilde{w}(y ; z)$. A way to prove this conjecture consists in studying precisely, via Theorem 1 , the singularities of $Q(x, y ; z)$.

\begin{tabular}{|c|c|c|c|c|}
\hline Group & Covariance & Walks & Nature of $Q$ & Nature of $w$ and $\widetilde{w}$ \\
\hline 4 & $=0$ & and 14 others [6] & $\begin{array}{l}\text { holonomic } \\
{[6,10]}\end{array}$ & $\begin{array}{c}\text { rational } \\
\text { [Theorems } 2 \text { and } 3 \text { (i)] }\end{array}$ \\
\hline 6 & $<0$ & 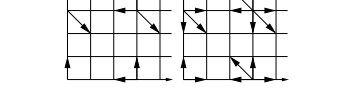 & $\begin{array}{l}\text { holonomic } \\
{[6,10]}\end{array}$ & $\begin{array}{c}\text { rational } \\
\text { [Theorems } 2 \text { and 3(ii)] }\end{array}$ \\
\hline 8 & $<0$ & 1. & $\begin{array}{l}\text { holonomic } \\
{[6,10]}\end{array}$ & $\begin{array}{c}\text { rational } \\
\text { [Theorems } 2 \text { and 3(iv)] }\end{array}$ \\
\hline 6 & $>0$ & 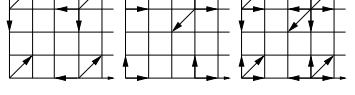 & $\begin{array}{c}\text { algebraic } \\
{[6,10]}\end{array}$ & $\begin{array}{c}\text { algebraic } \\
\text { [Theorems } 2 \text { and 3(iii)] }\end{array}$ \\
\hline 8 & $>0$ & 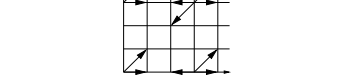 & $\begin{array}{c}\text { algebraic } \\
{[4,10]}\end{array}$ & $\begin{array}{c}\text { algebraic } \\
\text { [17] }\end{array}$ \\
\hline$\infty$ & $\begin{array}{l}=0 \\
<0 \\
>0\end{array}$ & and 50 others [6] & ? & $\begin{array}{l}\text { non-holonomic } \\
\text { [Theorem 2] }\end{array}$ \\
\hline
\end{tabular}

Fig. 6. Comparison a posteriori between the classification of the 74 non-singular walks according to the nature of the CGFs $w$ and $\widetilde{w}$ and the classification (still incomplete) according to the nature of the series $Q$, obtained from $[4,6,10]$. 
Acknowledgments. I would like to thank the members of the research team Algorithms of INRIA Rocquencourt, as well as Guy Fayolle, for the interesting discussions we had together regarding the topic of this article. I also would like to thank Irina Kurkova for her constant help and her encouragement all along the elaboration of this work. I thank Alexis Chommeloux for his numerous remarks about the English. Finally, I must thank two anonymous referees for their so careful reading and their valuable comments and suggestions.

\section{References}

[1] Adan, I., van Leeuwaarden, J., Raschel, K.: The compensation approach for walks with smallsteps in the quarter plane. Preprint (2011)

[2] Bostan, A., Chyzak, F., Kauers, M., Pech, L., van Hoeij, M.: Computing walks in a quadrant: a computer algebra approach via creative telescoping. In preparation (2011)

[3] Bostan, A., Kauers, M.: Automatic classification of restricted lattice walks. In: 21st Int. Conference on Formal Power Series and Algebraic Combinatorics, 201-215 (2009) MR 2721513

[4] Bostan, A., Kauers, M.: The complete generating function for Gessel walks is algebraic. Proc. Amer. Math. Soc. 432, 3063-3078 (2010) Zbl 1206.05013 MR 2653931

[5] Bousquet-Mélou, M.: Walks in the quarter plane: Kreweras' algebraic model. Ann. Appl. Probab. 15, 1451-1491 (2005) Zbl 1064.05010 MR 2134111

[6] Bousquet-Mélou, M., Mishna, M.: Walks with small steps in the quarter plane. In: Algorithmic Probability and Combinatorics, Contemp. Math. 520, Amer. Math. Soc., 1-40 (2010) Zbl 1209.05008 MR 2681853

[7] Bousquet-Mélou, M., Petkovšek, M.: Walks confined in a quadrant are not always D-finite. Theoret. Comput. Sci. 307, 257-276 (2003) Zbl 1070.68112 MR 2022578

[8] Chen, W., Deng, E., Du, R., Stanley, R., Yan, C.: Crossings and nestings of matchings and partitions. Trans. Amer. Math. Soc. 359, 1555-1575 (2007) Zbl 1108.05012 MR 2272140

[9] Fayolle, G., Iasnogorodski, R., Malyshev, V.: Random Walks in the Quarter-Plane. Springer, Berlin (1999) Zbl 0932.60002 MR 1691900

[10] Fayolle, G., Raschel, K.: On the holonomy or algebraicity of generating functions counting lattice walks in the quarter-plane. Markov Process. Related Fields 16, 485-496 (2010) Zbl pre05983783 MR 2759770

[11] Flajolet, P., Sedgewick, R.: Analytic Combinatorics. Cambridge Univ. Press, Cambridge (2009) Zbl 1165.05001

[12] Flatto, L.: Two parallel queues created by arrivals with two demands II. SIAM J. Appl. Math. 45, 861-878 (1985) Zbl 0579.90033 MR 0804012

[13] Flatto, L., Hahn, S.: Two parallel queues created by arrivals with two demands I. SIAM J. Appl. Math. 44, 1041-1053 (1984) Zbl 0554.90041 MR 0759714

[14] Gessel, I.: A probabilistic method for lattice path enumeration. J. Statist. Plann. Inference 14, 49-58 (1986) Zbl 0602.05006 MR 0845914

[15] Jones, G., Singerman, D.: Complex Functions. Cambridge Univ. Press, Cambridge (1987) Zbl 0608.30001 MR 0890746

[16] Kauers, M., Koutschan, C., Zeilberger, D.: Proof of Ira Gessel's lattice path conjecture. Proc. Nat. Acad. Sci. USA 106, 11502-11505 (2009) Zbl 1203.05010 MR 2538821

[17] Kurkova, I., Raschel, K.: Explicit expression for the generating function counting Gessel's walks. Adv. Appl. Math. 47, 414-433 (2011) Zbl pre05955931 MR 2822196

[18] Kurkova, I., Raschel, K.: Random walks in $\mathbb{Z}_{+}^{2}$ with non-zero drift absorbed at the axes. Bull. Soc. Math. France 139, 341-387 (2011) 
[19] Litvinchuk, G.: Solvability Theory of Boundary Value Problems and Singular Integral Equations with Shift. Kluwer, Dordrecht (2000) Zbl 0980.45001 MR 1885495

[20] Malyshev, V.: Positive random walks and Galois theory. Uspekhi Mat. Nauk 26, no. 1, $227-$ 228 (1971) (in Russian) Zbl 0254.60043 MR 0293729

[21] Mishna, M., Rechnitzer, A.: Two non-holonomic lattice walks in the quarter plane. Theor. Comput. Sci. 410, 3616-3630 (2009) Zbl pre05602724 MR 2553316

[22] Raschel, K.: Paths confined to a quadrant. PhD Thesis, Pierre et Marie Curie Univ. (2010)

[23] Raschel, K.: Green functions for killed random walks in the Weyl chamber of Sp(4). Ann. Inst. H. Poincaré Probab. Statist. 47, 1001-1019 (2011)

[24] Watson, G., Whittaker, E.: A Course of Modern Analysis. Cambridge Univ. Press, Cambridge (1962) Zbl 0105.26901 MR 0178117 